Article

\title{
Non-Equilibrium Liouville and Wigner Equations: Moment Methods and Long-Time Approximations
}

\author{
Ramon F. Álvarez-Estrada \\ Departamento de Fisica Teorica I, Facultad de Ciencias Fisicas, Universidad Complutense, \\ Madrid 28040, Spain; E-Mail: rfa@ucm.es; Tel.: +34-91-3944595
}

Received: 12 December 2013; in revised form: 7 February 2014 / Accepted: 24 February 2014 / Published: 11 March 2014

\begin{abstract}
We treat the non-equilibrium evolution of an open one-particle statistical system, subject to a potential and to an external "heat bath" $(h b)$ with negligible dissipation. For the classical equilibrium Boltzmann distribution, $W_{c, e q}$, a non-equilibrium three-term hierarchy for moments fulfills Hermiticity, which allows one to justify an approximate long-time thermalization. That gives partial dynamical support to Boltzmann's $W_{c, e q}$, out of the set of classical stationary distributions, $W_{c, s t}$, also investigated here, for which neither Hermiticity nor that thermalization hold, in general. For closed classical many-particle systems without $h b$ (by using $W_{c, e q}$ ), the long-time approximate thermalization for three-term hierarchies is justified and yields an approximate Lyapunov function and an arrow of time. The largest part of the work treats an open quantum one-particle system through the non-equilibrium Wigner function, $W . W_{e q}$ for a repulsive finite square well is reported. $W^{\prime}$ 's $(<0$ in various cases) are assumed to be quasi-definite functionals regarding their dependences on momentum $(q)$. That yields orthogonal polynomials, $H_{Q, n}(q)$, for $W_{e q}$ (and for stationary $W_{s t}$ ), non-equilibrium moments, $W_{n}$, of $W$ and hierarchies. For the first excited state of the harmonic oscillator, its stationary $W_{s t}$ is a quasi-definite functional, and the orthogonal polynomials and three-term hierarchy are studied. In general, the non-equilibrium quantum hierarchies (associated with $W_{e q}$ ) for the $W_{n}$ 's are not three-term ones. As an illustration, we outline a non-equilibrium four-term hierarchy and its solution in terms of generalized operator continued fractions. Such structures also allow one to formulate long-time approximations, but make it more difficult to justify thermalization. For large thermal and de Broglie wavelengths, the dominant $W_{e q}$ and a non-equilibrium equation for $W$ are reported: the non-equilibrium hierarchy could plausibly be a three-term one and possibly not far from Gaussian, and thermalization could possibly be justified.
\end{abstract}


Keywords: Liouville and Wigner distributions and non-equilibrium equations; equilibrium solutions and orthogonal polynomials; non-equilibrium moments; long-time approximations; low temperature regime

PACS Classification: $05.20 .-\mathrm{y}, 05.10 . \mathrm{Gg}, 05.20 . J \mathrm{~J}$, 05.30.-d, 05.30.Jp

\section{Introduction}

A large and comprehensive set of references on the philosophy and foundations of statistical mechanics (both at equilibrium and off-equilibrium), from different points of view, is collected in [1]. Non-equilibrium statistical systems of classical particles are described by non-negative Liouville distribution functions $\left(W_{c}\right)$ [2-5]. For non-equilibrium statistical systems of quantum particles, Wigner functions $(W)$ are quite suitable in a global sense [3-8] ( $W<0$ in some cases). Achieving a deeper knowledge of how open or closed (classical or quantum) statistical interacting systems evolve in time plays a key role in statistical physics. A system subject to the influence of another (in general, larger) system at thermal equilibrium (external "heat bath" or $h b$ ) is named open. A system not influenced by any such external $h b$ is named closed. The issue of the thermodynamic asymmetry in time continues to be central [9]: in short, how an arrow of time and/or irreversibility could arise in the long-time evolution of closed classical or quantum many-particle systems [2-5,10].

The dependences of the non-equilibrium Liouville and Wigner equations on momenta have a universal character (see Equations (1), (40) and (41)), while their dependences on positions are contained inside the potentials and, then, vary from one case to another. These facts suggest the use of non-equilibrium position-dependent moments (through suitable orthogonal polynomials profiting from the universal dependences on momenta and integrations over the latter). One could ask whether those universal momenta dependences would allow, for large systems under certain conditions and in some approximation, for their thermalization towards the canonical equilibrium distributions.

We shall adopt the following standpoint, certainly different from those in [11-14] and bearing connections to and, simultaneously, differences from those in [15-22]. As a direct analysis of large systems is very difficult, we shall start with an open small statistical system, subject to an $h b$ at thermal equilibrium at absolute temperature, $T(\neq 0)$, but with negligible external dissipation, due to the $h b$. In such a framework, the non-equilibrium evolution of the system does not thermalize, but we shall apply suitable long-time approximations and investigate whether they yield an approximate thermalization or if the latter is physically unexpected. Even if such dissipationless systems constitute an oversimplification, they give rise to both considerable difficulties and some interesting possibilities, as we shall see. Just in case an approximate thermalization could occur under certain conditions, then such a strategy could possibly be generalized for non-equilibrium evolutions of closed statistical many-particle systems without $h b$, at least approximately.

We shall study the following issues, left open in [11-14]: (a) In the non-equilibrium evolution for an open classical one-particle system for long times, subject to an external $h b$ at absolute temperature $T$ with negligible external dissipation on the former, is there anything in the structure of its Liouville equation 
allowing for certain dynamical selection of the canonical equilibrium distribution, out of the set of all stationary distributions, at least partially and/or approximately? If so, in what sense? If (a) has some positive (even if partial) answer: (b) could it be extended to non-equilibrium closed classical systems without external $h b$ (the role of the latter being then played by a large set of internal degrees of freedom at equilibrium in the system ), at least approximately? (c) Could it be extended to quantum systems by means of Wigner functions [6], at least approximately or in some regime? The present work, which will extend, non-trivially, [15-22], is devoted to study questions (a), (b) and (c).

First, we shall investigate Issue (a). See Section 2 (which will also provide a useful framework for (c) later, in Sections 3-7) and Appendices A and B.

Second (Issue (b)), we shall present a simple overview of [15] on long-time approximations and irreversibility in closed interacting many-particle classical systems (with neither $h b$ 's nor dissipation due to external sources), including some improvements from [17], in order to extract some approximate arrow of time. See Section 3 and Appendix B.

Third, we turn to the largest part of this work (Issue (c)). The genuine difficulties of quantum cases already show up in one-dimensional systems, and they deserve further study. Some generalizations of the moment method to non-equilibrium open one-particle quantum statistical systems have already been studied with external dissipation (to lowest order in Planck's constant) [21,22] and also without external dissipation [20]. Here, we shall focus on the Wigner function and equation, with negligible external dissipation due to the $h b$, to all orders in Planck's constant. We shall treat stationary and equilibrium Wigner functions, the issue of negativity and the orthogonal polynomials generated by them (Sections 4 and 5) and the non-equilibrium evolution (Section 6). Could one display some arrow of time in the quantum case, at least approximately in some regime? See Section 6.4 and, just for the case of large thermal and de Broglie wavelengths, Section 7. Finally, Section 8 presents some conclusions and various discussions. Several technical aspects on Issues (a) and (c) are treated in Appendices A and $\mathrm{C}-\mathrm{G}$.

The inclusion of non-negligible dissipation in open systems has been studied in detail, both in classical and quantum cases, in [22]. From the outset, in the present work, we shall deal with an open system, subject to an $h b$ (at finite and well-defined $T \neq 0$ ) exerting negligible dissipation on the former. However, the very consideration of such a system could give rise to criticisms. In fact, one could argue that either the neglection of dissipation would upset the fact that the system evolves at finite $T$ or that the system would be a closed one ( then being unclear what the temperature would mean ). We shall address such conceptual issues a posteriori in Sections 2.3 (classical) and 6.4 (quantum).

\section{Open Classical One-Particle Systems}

\subsection{Some General Aspects}

Let a classical non-relativistic particle, with mass $m$, position $x$ and momentum $q$, be subject to a real potential $V=V(x)$, in the presence of a "heat bath" $(h b)$ at thermal equilibrium at absolute temperature $T$, with $\beta=\left(k_{B} T\right)^{-1}$ ( $k_{B}$ being Boltzmann's constant). Dissipation effects on the particle due to the $h b$ will be supposed as so small that they will be discarded completely (negligible friction). 
We shall suppose that $V(x) \geq 0$, with $V(x) \rightarrow 0$ as $|x| \rightarrow+\infty$. Classical harmonic/anharmonic oscillators, treated in [17,20], will not be considered in Sections 2 and 3. The classical Hamiltonian of the particle is: $H_{c}=q^{2} /(2 m)+V$. Let the classical particle be, at the initial time $t=0$, out of thermal equilibrium with the $h b$, and have a Liouville probability distribution $W_{c, i n}=W_{c, i n}(x, q)(\geq 0)$ to be at the position, $x$, with momentum $q$. Then, the non-equilibrium particle has, at time $t(>0)$, the probability distribution $W_{c}=W_{c}(x, q ; t)(\geq 0)$, which evolves through the reversible Liouville equation:

$$
\frac{\partial W_{c}}{\partial t}+\frac{q}{m} \frac{\partial W_{c}}{\partial x}-\frac{\partial V}{\partial x} \frac{\partial W_{c}}{\partial q}=0
$$

We shall treat the temporal evolution for $t>0$ by using Equation (1) for $W_{c}$, with the initial condition, $W_{c, i n}$. Equation (1) has an infinite set of stationary ( $t$-independent) solutions: $W_{c}=W_{c, s t}=f\left(H_{c}\right)$, with an arbitrary function, $f \geq 0$, decreasing quickly as $\left|H_{c}\right| \rightarrow+\infty$, by assumption. Hence, $W_{c, s t}$ is not Gaussian in $q$, in general. The equilibrium (or Boltzmann's) canonical distribution, a $t$-independent solution of Equation (1) describing thermal equilibrium of the particle with the $h b$, is Gaussian in $q$ : $W_{c, s t}=W_{c, e q}=\exp \left[-\beta\left(q^{2} /(2 m)+V\right)\right]$. Another stationary solution, to be treated briefly in Appendix B, just as a peculiar illustration, is the stationary microcanonical distribution, $W_{c, m c}$.

Let $q_{0}$ be some fixed $x$-independent momentum, to be discussed later. We shall define $y=q / q_{0}$. We shall consider a generic stationary $W_{c, s t}$, with an arbitrary $f$, and we shall introduce the denumerably infinite family of all (unnormalized) polynomials in $y: H_{c, n}=H_{c, n}(y)(n=0,1,2,3, \ldots)$, orthogonalized in $y$ (for fixed $x$ ) by using $W_{c, s t}$ as the weight function. By choosing $H_{c, 0}(q)=1$, we impose, for $n \neq n^{\prime}$ and any $x$ (left unintegrated), that:

$$
\int_{-\infty}^{+\infty} d y W_{c, s t}(x, q) H_{c, n}(y) H_{c, n^{\prime}}(y)=0
$$

The orthonormalized polynomials are $H_{c, n}(y) /\left(h_{c, n}\right)^{1 / 2}$, with the ( $x$-dependent) normalization factor:

$$
h_{c, n} \equiv \int_{-\infty}^{+\infty} d y W_{c, s t}(x, q) H_{c, n}(y)^{2}
$$

The $H_{c, n}(y)$ 's suggest the following new moments $W_{c, n}(n=0,1,2, \ldots)$ of $W_{c}$ :

$$
W_{c, n}=W_{c, n}(x ; t)=\int_{-\infty}^{+\infty} d y H_{c, n}(y) W_{c}(x, q ; t)
$$

For $W_{c}=W_{c, s t}(x, q)$, Equation (4) yields $W_{c, s t, n}=0$ if $n>0$, and $W_{c, s t, 0}=\int_{-\infty}^{+\infty} d y W_{c, s t}(x, q)$. The initial condition, $W_{c, i n, n}$, for $W_{c, n}$ is obtained by replacing $W_{c}$ by $W_{c, i n}$ in Equation (4). One has the following (formal) expansion for $W_{c}$ :

$$
W_{c}=W_{c, s t}(x, q) \sum_{n=0}^{+\infty} W_{c, n}(x ; t) \frac{H_{c, n}(y)}{h_{c, n}}
$$

2.2. $W_{c, s t}=W_{c, e q}$ : Three-Term Hierarchy, Operator Continued Fractions and Long-Time Approximation

Let us consider the very special case: $W_{c, s t}=W_{c, e q}$, with $q_{0}=(2 m / \beta)^{1 / 2}$. The orthogonal polynomials generated by the weight function, $W_{c, e q}$, are $H_{c, n}(y)=H_{n}(y), H_{n}(y)$ being the standard 
( $x$-independent) Hermite polynomials [23]. For $W_{c}=W_{c, e q}(x, q)$ and $H_{c, n}(y)=H_{n}(y)$, Equation (4) yields $W_{c, e q, n}=0$ if $n>0$, and $W_{c, e q, 0}=\int_{-\infty}^{+\infty} d y W_{c, e q}(x, q)=\pi^{1 / 2} \exp [-\beta V]$. For $H_{c, n}(y)=H_{n}(y)$, Equations (1) and (4) give an exact three-term non-equilibrium hierarchy for all $W_{c, n}$ 's $[11,12,15]$. Such a three-term hierarchy, through the transformation $W_{c, n}(x ; t)=\left[W_{c, e q, 0}(x)\right]^{1 / 2} g_{n}(x ; t)$, becomes the following exact three-term hierarchy for the $g_{n}$ 's for any $n=0,1,2,3 \ldots$ :

$$
\begin{aligned}
& \frac{\partial g_{n}}{\partial t}=-M_{c, n, n+1} g_{n+1}-M_{c, n, n-1} g_{n-1} \\
& M_{c, n, n \pm 1} g_{n \pm 1} \equiv\left[\frac{(n+(1 / 2)(1 \pm 1)) k_{B} T}{m}\right]^{1 / 2}\left[\frac{\partial g_{n \pm 1}}{\partial x}-\frac{( \pm 1) g_{n \pm 1}}{2 k_{B} T} \frac{\partial V}{\partial x}\right]
\end{aligned}
$$

with initial condition $g_{i n, n}=W_{c, i n, n} / W_{c, e q, 0}^{1 / 2}$. One key fact is that $M_{c, n, n+1}$ and $-M_{c, n+1, n}$ are the adjoint of each other. Notice that Equation (7) corrects a misprint in Equation (5) in [20]. One sees directly that the $W_{c, e q, n}$ 's solve the three-term recurrence relation Equations (6) and (7).

Let us consider the Laplace transforms $\tilde{g}_{n}=\tilde{g}_{n}(s)=\int_{0}^{+\infty} d t g_{n} \exp (-s t)$, with inverse $g_{n}=\int_{c-i \infty}^{c+i \infty}(d s / 2 \pi i) \tilde{g}_{n} \exp (s t)$ ( $c$ being real and such that $\tilde{g}_{n}(s)$ is analytic in the half-plane Res $>c$ of the complex $s$-plane). This definition and Equation (6) yield the three-term hierarchy for $\tilde{g}_{n}$ :

$$
s \tilde{g}_{n}=g_{i n, n}-M_{c, n, n+1} \tilde{g}_{n+1}(s)-M_{c, n, n-1} \tilde{g}_{n-1}(s)
$$

The hierarchy Equation (8) can be solved formally by extending to it standard procedures for solving numerical three-term linear recurrence relations in terms of continued fractions [12]. One gets all $\tilde{g}_{n}(s)$, for any $n=1, \ldots$, in terms of sums of products of certain $s$-dependent linear operators $D\left[n^{\prime} ; s\right], n^{\prime} \geq n$, acting upon $\tilde{g}_{n-1}(s)$ and upon all $g_{i n, n^{\prime}}$ 's, with $n^{\prime} \geq n$. The following recurrence gives the $D[n ; s]$ 's:

$$
D[n ; s]=\left[s I-M_{c, n, n+1} D[n+1 ; s] M_{c, n+1, n}\right]^{-1}
$$

$I$ is the unit operator. By iteration of Equation (9), $D[n ; s]$ becomes a formal infinite continued fraction of the products of the non-commuting linear operators, $M_{c, n, n+1}$ and $M_{c, n+1, n}$. The hierarchies in Equations (6)-(8) are as reversible as Equation (1). The long-time approximation below will introduce irreversibility in those hierarchies. Let us consider some real and small $\epsilon>0$ and some integer, $n_{0} \geq 1$. Then, for $n \geq n_{0}, D[n+1 ; \epsilon]$ is Hermitian, and all its eigenvalues are real and non-negative. The essential justification of those two properties can be easily exemplified through $2 \times 2$ matrices: let us replace $D[n+1 ; s]$ by a Hermitian $2 \times 2$ matrix with non-negative eigenvalues, $M_{c, n, n+1}$ and $M_{c, n+1, n}$, by two $2 \times 2$ matrices, such that $M_{c, n, n+1}$ and $-M_{c, n+1, n}$ are the adjoint of each other. Then, it is easy to see that $-M_{c, n, n+1} D[n+1 ; s] M_{c, n+1, n}$ is also a Hermitian $2 \times 2$ matrix with non-negative eigenvalues, and the same holds for $\epsilon I-M_{c, n, n+1} D[n+1 ; s] M_{c, n+1, n}, I$ being now the unit $2 \times 2$ matrix. An alternative justification of the above is also given just before Equation (32). To complete the justification, one should take into account the $\left.\left[(n+(1 / 2)(1 \pm 1)) k_{B} T\right)\right]^{1 / 2}$ factors contained inside $M_{c, n, n \pm 1}$ in Equation (7): the continued-fraction structure of the $D$ 's in Equation (9) implies that for adequately large $n,-M_{c, n, n+1} D[n+1 ; s] M_{c, n+1, n}$ decreases grossly as $n^{-1 / 2}$, so that, if $\epsilon$ is not large, $\left[\epsilon I-M_{c, n, n+1} D[n+1 ; s] M_{c, n+1, n}\right]^{-1}$ is also Hermitian and has non-negative eigenvalues. In short, the continued-fraction structure of the $D$ 's in Equation (9) justifies recurrently that if $D[n+1 ; \epsilon]$ is Hermitian and has only non-negative eigenvalues, the same is also true for $D[n ; \epsilon]$. For more details, 
see $[15,16,20]$. For a simpler hierarchy, without loss of generality, let us assume that $W_{c . i n, n^{\prime}}=0$ for $n^{\prime} \geq 1$, with $W_{c, i n, 0} \neq 0$. Although the long-time approximation has been justified for adequately large $n$, let us choose $n_{0}=1$, for simplicity. Then, Equation (8) yields:

$$
\begin{aligned}
& s \tilde{g}_{0}(s)=W_{c, e q, 0}^{-1 / 2} W_{c, i n, 0}-M_{c, 0,1} \tilde{g}_{1}(s) \\
& \tilde{g}_{n}(s)=-D[n ; s] M_{c, n, n-1} \tilde{g}_{n-1}(s), n \geq 1
\end{aligned}
$$

The long-time approximation for $n \geq 1$ and small $s$ can now be implemented as follows (with $\epsilon>0$ ). One replaces any $D[n ; s]$ yielding $\tilde{g}_{n}$ in Equation (11), $n \geq 1$, by $D[n ; \epsilon]$ : this approximation is not done for $n=0$, which will be crucial. We regard $D[n ; \epsilon]$ in Equation (11) as fixed (s-independent) operators. Thus, in particular, we approximate in Equation (11) for small $s$ and $n=1$ as: $\tilde{g}_{1}(s) \simeq-D[1 ; \epsilon] M_{c, 1,0} \tilde{g}_{0}(s)$ (to be compared to Equation (11) for $n=1$, before the approximation). Then, Equations (11) (after the approximation) and (10) become, by taking inverse Laplace transforms:

$$
\begin{aligned}
& \frac{\partial g_{0}}{\partial t}=-M_{c, 0,1} g_{1} \\
& g_{1} \simeq-D[1 ; \epsilon] M_{c, 1,0} g_{0}
\end{aligned}
$$

Equations (12) and (13) give the irreversible Smoluchowski-like equation for the $n=0$ moment:

$$
\partial g_{0} / \partial t=M_{c, 0,1} D[1 ; \epsilon] M_{c, 1,0} g_{0}
$$

In the latter, after the above long-time approximation, we employ the same initial condition $W_{c, i n, 0} / W_{c, e q, 0}^{1 / 2}$ at $t=0$; this amounts to another kind of approximation (presumably, without special importance, as long as the system will thermalize, independently on the initial condition). The solutions of the last equation for $g_{0}$ relax irreversibly, for large $t$ and reasonable $W_{c, i n, 0}$, towards $W_{c, e q, 0}^{1 / 2} \neq 0$ and $g_{n} \rightarrow 0$ if $n=1, \ldots$ (thermal equilibrium). Then, the dominant moment is $g_{0}$, while any $g_{n}$ with $n>0$ is negligible. $g_{n}(n \geq 1)$ is the smaller, the larger $t(>0)$ and $n$ are (due to the behaviors of $M_{c, n, n \pm 1}$ and of $D[n ; \epsilon]$ with $n)[15,16,20]$. Similar behaviors hold for $W_{c, 0}$ and $W_{c, n}$ with $n>0$. To carry out quantitative studies for large $t$, some ansatz or approximation (outside our scope) should be provided for $D[1 ; \epsilon]$ at least, consistently with the above properties. Equation (102) describes a Brownian-like random walker, subject to $V(x)$. For other applications of the operator continued fractions to the Smoluchowski equation for Brownian motion and to problems in condensed matter physics, see [24] and references therein.

For the following computation, the right-hand-side of Equation (14) should be interpreted as $\int_{-\infty}^{+\infty} d x^{\prime}\left(M_{c, 0,1} D[1 ; \epsilon] M_{c, 1,0}\right)\left(x, x^{\prime}\right) g_{0}\left(x^{\prime} ; t\right)$. Let: $\left(f_{1}, f_{2}\right)=\int_{-\infty}^{+\infty} d x f_{1}(x)^{*} f_{2}(x)$ for suitable functions $f_{1}$ and $f_{2}$. The following properties (which follow from the general ones of $D[n ; \epsilon]$ stated above) will be used to get Equation (16): (a) due to the Hermiticity of $D[1 ; \epsilon]$ : $\left(f_{1}, M_{c, 0,1} D[1 ; \epsilon] M_{c, 1,0} f_{2}\right)=\left(M_{c, 0,1} D[1 ; \epsilon] M_{c, 1,0} f_{1}, f_{2}\right)$, thereby checking that $M_{c, 0,1} D[1 ; \epsilon] M_{c, 1,0}$ is Hermitian, as so is $D[1 ; \epsilon]$; (b) $\left(f_{1}, M_{c, 0,1} D[1 ; \epsilon] M_{c, 1,0} f_{1}\right)=-\left(M_{c, 1,0} f_{1}, D[1 ; \epsilon] M_{c, 1,0} f_{1}\right) \leq 0$ for arbitrary functions $f_{1}$, as all eigenvalues of $D[1 ; \epsilon]$ are $\geq 0$. Both Properties (a) and (b) (with $D[1 ; \epsilon]$ replaced by $D[n+1 ; \epsilon]$ ) lie at the heart of the iterative justification that $D[n ; \epsilon]$ is Hermitian and has only real and non-negative eigenvalues for $n \geq 0$. After the long-time approximation and anticipating Equation (32), let:

$$
L=\frac{1}{2}\left(g_{0}, g_{0}\right)
$$


Using Equation (14) and the preceding Properties (a) and (b):

$$
\partial L / \partial t=\left(g_{0}, M_{c, 0,1} D[1 ; \epsilon] M_{c, 1,0} g_{0}\right)=-\left(M_{c, 1,0} g_{0}, D[1 ; \epsilon] M_{c, 1,0} g_{0}\right) \leq 0
$$

As $L$ is a non-decreasing function in the time evolution, it can be regarded as a Lyapunov function. A function quite similar to this $L$ has been studied for the Brownian motion of one classical particle [25] (but without having arrived to it, by starting from Equation (1)).

\section{3. $W_{c, s t}=W_{c, e q}:$ On the Inclusion of Dissipation}

The following discussion on dissipation is adequate. We do not attribute a physical meaning to temperature for a closed classical one-particle system (contrary to a closed classical many-particle one, to be treated in Section 3). There is a physical difference between a closed classical one-particle system and the meaningful open one in the presence of (and coupled to) an external $h b$ at thermal equilibrium at finite $T$, with friction. By recalling [20], we shall treat briefly the inclusion of non-negligible dissipation with friction constant $\sigma>0$ for the open classical system. Then, Equations (1), (8) and (9) are replaced, respectively, by:

$$
\begin{aligned}
& \frac{\partial W_{c}}{\partial t}+\frac{q}{m} \frac{\partial W_{c}}{\partial x}-\frac{\partial V}{\partial x} \frac{\partial W_{c}}{\partial q}=\frac{1}{\sigma} \frac{\partial\left[(q+(m / \beta))\left(\partial W_{c} / \partial q\right)\right]}{\partial q} \\
& \left(s+\frac{n}{\sigma}\right) \tilde{g}_{n}=W_{c, e q, 0}^{-1 / 2} W_{c, i n, n}-M_{n, n+1} \tilde{g}_{n+1}-M_{n, n-1} \tilde{g}_{n-1} \\
& D\left[n ; s+\frac{n}{\sigma}\right]=\left[s+\frac{n}{\sigma}-M_{n, n+1} D\left[n+1 ; s+\frac{n+1}{\sigma}\right] M_{n+1, n}\right]^{-1}
\end{aligned}
$$

Equation (17) is a well-known dissipative Equation [11-13]. Notice the key fact that in Equations (18) and (19), $\sigma$ only appears as $s+(n / \sigma)$, so that $\epsilon+(n / \sigma)>0$ (for $s=\epsilon>0$ and $n=0,1,2, \ldots$ ). Having in mind Equation (7) (and the $T$-dependences in it), one can let $\sigma^{-1}$ be as small as one wishes at constant and finite $T$, while $D\left[n ; \epsilon+\frac{n}{\sigma}\right]$ always fulfills Hermiticity and non-negativity, regardless of how its magnitude could vary (which, in turn, depends on $\sigma^{-1}, n, \epsilon$ and $T$, for given $V$ ). This is the essential argument allowing one to use a $h b$ at finite $T$ with very small dissipation and in trying to justify that the step $\sigma^{-1} \simeq 0$ could be permissible (those properties of $D\left[n ; \epsilon+\frac{n}{\sigma}\right]$ not depending on whether $\sigma^{-1}$ is very small, with $\neq 0$, or $\sigma^{-1} \simeq 0$ ), in the classical case. However, just for $\sigma^{-1} \simeq 0$, one could argue that the resulting $\sigma^{-1}$-free equations (namely, Equations (1), (8) and (9), containing a finite $T$ ) account for a closed classical one-particle system. We shall not delve into the conceptual issue of whether the step $\sigma^{-1} \simeq 0$ allows for the classical one-particle system in the presence of an $h b$ at finite $T$ to be still interpreted as open (which we have favored and employed) or leads to a closed one. We can avoid that issue for the following practical reason: (oversimplified) open classical one-particle systems are interesting, as long as they provide useful technical guides towards approximate long-time thermalization in non-equilibrium closed classical large systems (without external $h b$ 's), and that will turn out to be the case in Section 3.

A similar practical standpoint in the (far more difficult) quantum case will be expressed in the last paragraph in Section 6.4. 


\subsection{Generic $W_{c, s t}$ : Hierarchy and Failures of the Long-Time Approximation}

For a general $W_{c, s t}$, with given ( $x$-independent) $q_{0}$, we shall return to the $H_{c, n}$ 's defined in Equation (2) (depending parametrically on $x$ in general for $n \geq 2$ ), so that $H_{c, n} \neq H_{n}$. We shall write them as:

$$
H_{c, n}(y)=y^{n}+\sum_{j=1}^{n} \epsilon_{c, n, n-j} y^{n-j}
$$

$\epsilon_{c, n, n-j}$, being $y$-independent (but $x$-dependent, in general), is given in Appendix A for $n=2,3,4,5$ (Equations (73)-(76)). As $W_{c, s t}$ is even in $y$, one has $\epsilon_{c, n, n-j}=0$ for odd $j$, so that $H_{c, n}(-y)=(-1)^{n} H_{c, n}(y)$. Consistently with the general properties [26], the $H_{c, n}$ 's fulfill the three-term recurrence relation:

$$
y H_{c, n}(y)=H_{c, n+1}(y)+C_{c, n} H_{c, n-1}(y)
$$

for $n=0,1,2, \ldots$, with $C_{c, 0} \equiv 0 . C_{c, n}$ is $y$-independent (but it depends on $x$ ). Since $W_{c, s t} \geq 0$, a general theorem [26] ensures that $C_{c, n} \geq 0$. Examples of the lowest $C_{c, n}$ 's for the stationary microcanonical distribution are given in Appendix B. One could also express the $H_{c, n}$ 's as suitable sums of Hermite polynomials: such an alternative will not be followed here, but in Section 5, in other cases.

The $H_{c, n}$ 's determine new moments $W_{c, n}$ (not to be confused with those in Section 2.2 for the $H_{n}$ 's) through Equation (4). We shall transform Equation (1) into a linear hierarchy for the new moments, $W_{c, n}$. For that purpose, we multiply Equation (1) by $H_{c, n}(y)$, integrate over $y$ and operate, so as to express the resulting equation solely in terms of the $W_{c, n}$ 's. We carry out cancellations and simplifications, by using Equations (1) and (20). We shall display the first five equations (for $n=0,1,2,3,4$ ) in the hierarchy, for any $W_{c, s t}$ and $q_{0}$ :

$$
\begin{aligned}
& \frac{\partial W_{c, 0}}{\partial t}=-\frac{q_{0}}{m} \frac{\partial W_{c, 1}}{\partial x} \\
& \frac{\partial W_{c, 1}}{\partial t}=-\frac{q_{0}}{m} \frac{\partial W_{c, 2}}{\partial x}+\frac{q_{0}}{m} \frac{\partial}{\partial x}\left[\left(\epsilon_{c, 2,0}\right) W_{c, 0}\right]-\frac{1}{q_{0}} \frac{\partial V}{\partial x} W_{c, 0} \\
& \frac{\partial W_{c, 2}}{\partial t}=-\frac{q_{0}}{m} \frac{\partial W_{c, 3}}{\partial x}+\frac{q_{0}}{m} \frac{\partial}{\partial x}\left[\left(\epsilon_{c, 3,1}-\epsilon_{c, 2,0}\right) W_{c, 1}\right]+\frac{q_{0}}{m} \frac{\partial \epsilon_{c, 2,0}}{\partial x} W_{c, 1}-\frac{2}{q_{0}} \frac{\partial V}{\partial x} W_{c, 1} \\
& \frac{\partial W_{c, 3}}{\partial t}=-\frac{q_{0}}{m} \frac{\partial W_{c, 4}}{\partial x}+\frac{q_{0}}{m} \frac{\partial}{\partial x}\left[\left(\epsilon_{c, 4,2}-\epsilon_{c, 3,1}\right) W_{c, 2}\right]+\frac{q_{0}}{m} \frac{\partial \epsilon_{c, 3,1}}{\partial x} W_{c, 2}-\frac{3}{q_{0}} \frac{\partial V}{\partial x} W_{c, 2} \\
& \frac{\partial W_{c, 4}}{\partial t}=-\frac{q_{0}}{m} \frac{\partial W_{c, 5}}{\partial x}+\frac{q_{0}}{m} \frac{\partial}{\partial x}\left[\left(\epsilon_{c, 5,3}-\epsilon_{c, 4,2}\right) W_{c, 3}\right]+\frac{q_{0}}{m} \frac{\partial \epsilon_{c, 4,2}}{\partial x} W_{c, 3}-\frac{4}{q_{0}} \frac{\partial V}{\partial x} W_{c, 3}
\end{aligned}
$$

Equations (77)-(80) play a key role in the computations and cancellations leading to Equations (22)-(26). The latter constitute a three-term recurrence relation for $0 \leq n \leq 4$. For any $W_{c, s t} \neq W_{c, e q}$ and $V \neq 0$, the equation for $\partial W_{c, n} / \partial t$ for $n \geq 5$ contains $M_{s t, n, n+1} W_{c, n+1}=\left(q_{0} / m\left(\partial W_{c, n+1} / \partial x\right.\right.$ and no other dependences on higher order moments, but it is increasingly difficult to exhibit cancellations in it for moments of order lower that $n$. Then, it is an open question whether the hierarchy Equations (22)-(26), when analyzed for $5 \leq n \leq+\infty$, is also a three-term one regarding moments of order lower than $n$. In spite of that, and for a generic $W_{c, s t}\left(\neq W_{c, e q}\right)$, let us conjecture, tentatively, for 
a while, that, for $V \neq 0$, the $W_{c, n}$ 's would fulfill an infinite three-term hierarchy, with other operators $M_{s t, n, n \pm 1}\left(\neq M_{c, n, n \pm 1}\right)$, namely:

$$
\frac{\partial W_{c, n}}{\partial t}=-M_{s t, n, n+1} W_{c, n+1}-M_{s t, n, n-1} W_{c, n-1}
$$

with the above $M_{s t, n, n+1}$. The corresponding $M_{s t, n, n-1}$ 's are linear first-order differential operators, and those for $n=0,1,2,3,4$ are obtained by comparison with Equations (22)-(26). That is, the finite hierarchy Equations (22)-(26) would be the lowest part of the infinite three-term one in Equation (27), conjectured to hold for any $5 \leq n \leq+\infty$. See Section 7 for one further development regarding such a conjecture. The initial conditions at $t=0$ continue to be $W_{c, i n, n}$. One sees directly that the stationary moments $W_{c, s t, n}$ 's do solve the three-term recurrence relations Equations (22)-(26), (27). A key issue for a generic $W_{c, s t}\left(\neq W_{c, e q}\right)$ is that $M_{s t, n, n+1}$ is not the adjoint of $-M_{s t, n+1, n}$ for any $n$ in general, as Equations (22)-(26) exemplify. Moreover, we have been unable to transform exactly the hierarchy Equation (27) into another one with new $W_{c, n}^{\prime}$ and $M_{s t, n, n \pm 1}^{\prime}$, in which $M_{s t, n, n+1}^{\prime}$ is the adjoint of $-M_{s t, n+1, n}^{\prime}$ for any $n$, and we believe that, likely, such a transformation does not exist in general.

Let us consider the Laplace transforms $\tilde{W}_{c, n}=\tilde{W}_{c, n}(s)=\int_{0}^{+\infty} d t W_{c, n} \exp (-s t)$. This definition yields an infinite three-term hierarchy for $\tilde{W}_{c, n}$ 's similar to Equation (8) which, in turn, can be solved formally in terms of other operator continued fractions, $D_{s t}[n ; s]$ 's. The latter are defined recurrently through:

$$
D_{s t}[n ; s]=\left[s I-M_{s t, n, n+1} D_{s t}[n+1 ; s] M_{s t, n+1, n}\right]^{-1}
$$

As $M_{s t, n, n+1}$ and $-M_{s t, n+1, n}$ are not adjoint to each other, it follows that $D_{s t}[n+1 ; \epsilon]$ is not Hermitian in general, and no general statement can be made regarding its eigenvalues, if they exist. Then, a priori, it is unlikely that a long-time approximation and a long-time approach to equilibrium for $n \geq n_{0} \geq 1$ could be justified for the $\tilde{W}_{c, n}$ 's in the three-term Equation (27), for an arbitrary $W_{c, s t}$. Upon recalling at this stage that the validity of the three-term structure in Equation (27) has not even been established if $V \neq 0$ for $5 \leq n \leq+\infty$, the possibility of formulating a long-time approximation for a generic $W_{c, s t}\left(\neq W_{c, e q}\right)$ becomes even more unlikely. It is unclear whether the microcanonical distribution $W_{c, s t}=W_{c, m c}$ could be an exception, somehow: see Appendix B.

Then, the developments in Section 2.2 and in the present one appear to indicate that, if $\epsilon>0$, there would exist some sort of (at least, partial) dynamical selection of the canonical equilibrium distribution, $W_{c, e q}(x, q)$, out of the set of all stationary distributions, $W_{c, s t}$, solving the Liouville equation. On the other hand, the general recurrence Equations (22)-(26) and the arguments on the $D_{s t}$ 's in this subsection will turn out to be very useful in Sections 6 and 7. 


\section{Closed Classical Many-Particle Systems: Long-Time Approximation and the Arrow of Time}

We treat a closed large system of many $(N \gg 1)$ classical non-relativistic particles, in $d$ spatial dimensions $(d=1,2,3)$, with spatial coordinates $\mathbf{x}_{1}, \ldots, \mathbf{x}_{N}(\equiv[x])$ and momenta $\mathbf{q}_{1}, \ldots, \mathbf{q}_{N}(\equiv[q])$ : a real gas. All particles, which are identical, have mass $m$. Let $x_{i, \alpha}$ and $q_{i, \alpha}$ be the Cartesian components of $\mathbf{x}_{i}$ and $\mathbf{q}_{i}$, respectively $(i=1, \ldots, N, \alpha=1, \ldots d)$. Neither a $h b$, nor external friction mechanisms, nor external forces are included. The interaction potential among all particles is: $V=\Sigma_{i, j=1, i<j}^{N} V_{i, j}\left(\left|\mathbf{x}_{i}-\mathbf{x}_{j}\right|\right)$. By assumption, all $V_{i, j}\left(\left|\mathbf{x}_{i}-\mathbf{x}_{j}\right|\right)$ are repulsive $(\geq 0)$ and tend quickly to zero for large $\left|\mathbf{x}_{i}-\mathbf{x}_{j}\right|$. The non-equilibrium classical distribution function is: $W_{c}=W_{c}([x],[q] ; t)$. The reversible Liouville equation reads:

$$
\frac{\partial W_{c}}{\partial t}=\Sigma_{i=1}^{N} \Sigma_{\alpha=1}^{d}\left[\frac{\partial V}{\partial x_{i, \alpha}} \frac{\partial W_{c}}{\partial q_{i, \alpha}}-\frac{q_{i, \alpha}}{m} \frac{\partial W_{c}}{\partial x_{i, \alpha}}\right]
$$

See [3,5,27] for studies of non-equilibrium states. The initial $W_{c, i n}[3,5,15]$, given by assumption, describes the following non-equilibrium distribution at $t=0$. There is a very large set $\left(s_{1}\right)$ of degrees of freedom in the system, which are at thermal equilibrium at temperature $T$ with one another, and they play the role of an effective (internal) $h b$. The gas at $t=0$ also contains a large set of degrees of freedom $\left(s_{2}\right)$, which are off-equilibrium with the previous set, $s_{1}$, and among themselves. We may suppose that the set, $s_{1}$, is located at large distances and is larger than the set, $s_{2}$ (located, in turn, at finite distances). We shall focus on Boltzmann's equilibrium distribution at temperature $T: W_{c, e q}=\exp \left[-\beta H_{c, N}\right]$, where $H_{c, N}=(2 m)^{-1} \sum_{i=1}^{N} \Sigma_{\alpha=1}^{d} q_{i, \alpha}^{2}+V$ is the classical $N$-particle Hamiltonian. Let $[n]$ denote a set of non-negative integers $(n(i=1, \alpha=1), \ldots, n(i=N, \alpha=d))$, and let $n=\Sigma_{l=1}^{N} \Sigma_{\alpha=1}^{d} n(l, \alpha)$. Let $[d q]=\prod_{i=1}^{N} \prod_{\alpha=1}^{d} d q_{i, \alpha}$.

We introduce non-equilibrium moments $W_{[n]}$ of $W$ (using products of the Hermite polynomials orthogonalized through $W_{c, e q}$, by generalizing Equation (4)):

$$
\int[d q] \prod_{i=1}^{N} \prod_{\alpha=1}^{d} \frac{H_{n(i, \alpha)}\left(q_{i, \alpha} /\left(2 m k_{B} T\right)^{1 / 2}\right)}{\left(\pi^{1 / 2} 2^{n(i, \alpha)} n(i, \alpha) !\right)^{1 / 2}} W_{c}([x],[q], t) \equiv W_{c}(x ;[n] ; t)=W_{c}([n])
$$

with the choice $q_{0}=(2 m / \beta)^{1 / 2}$. If $W_{c}=W_{c, e q}$, then $W_{c, e q}([0])=\pi^{3 N / 4} q_{0}^{3 N} \exp [-\beta V]$ $([0]=(0,0, \ldots, 0))$ and $W_{c, e q}([n])=0,[n] \neq[0](n>0)$. Equation (30) can also be applied to $W_{c, i n}$ and gives the corresponding initial moments, $W_{c, i n}([n])$. We shall work with the symmetrized moments $g([n])=W_{c, e q}([0])^{-1 / 2} W_{c}([n])$. The resulting infinite reversible three-term hierarchy for $g([n])$ 's (for any $[n]$, generalizing Equations (6) and (7)) and the long-time approximation and evolution towards $W_{c, e q}$, have been justified previously $[15,20]$. In principle, the long-time approximation also requires here a similar $\epsilon>0$; see [15,20] for the possibility of letting $\epsilon \rightarrow 0$ here (as $N$ is very large and $d>1$, in the thermodynamical limit). One expects that if $W_{c, i n}$ is not too far from $W_{c, e q}$, then the thermalization of the whole system at constant $T$ throughout it occurs. The arguments in Section 2.2, based upon Hermiticity and non-negativity and justifying partially the dynamical selection of $W_{c, e q}$, also hold in the present case; the details will be omitted. A posteriori, the formal similarity between Equations (6) and (7) and the actual three-term hierarchy for $N$ particles (with vanishing external dissipation) [15] justify the usefulness of the studies in Section 2 for $h b$ 's with negligible dissipation. 
Both the exact hierarchy for the $g([n])$ 's and the closed approximate one for them after the long-time approximation are genuinely different from the non-equilibrium classical BBGKY (Bogoliubov-BornGreen-Kirkwood-Yvon) hierarchy [2-4]. In the latter, in the equation for the distribution function for $n(>0)$ particles, one leaves unintegrated their position vectors and momenta, while those for the remaining $N-n$ particles are integrated over. Moreover, such an equation also depends on the distribution function for $n+1$ particles, but not on that for $n-1$ ones, a feature that, beyond the approximate framework of the standard Boltzmann equation (for $n=1$ ) [2-4], does not seem to shed much light on the long-time approach to thermal equilibrium for larger $n$. By contrast, in the equation for $g([n])$ in the actual non-equilibrium hierarchy based upon $W_{c, e q}$, the contributions from $g([n+1])$ 's are neatly different from (and, for large $n$, approximately smaller by a factor of $\simeq n^{-1 / 2}$ than) those coming from $g([n])$ 's, at least in the long-time approximation $[15,20]$.

As an example, let us consider the following irreversible Smoluchowski-like equation for the $[n=0]$ moment, which follows from long-time approximation [15,22]. It generalizes Equation (14) $([n=0]$ meaning $n(1,1)=0, \ldots, n(j, \beta)=0, \ldots, n(N, d)=0)$ :

$$
\begin{aligned}
& \frac{\partial g([n=0])}{\partial t}=\Sigma_{l=1}^{N} \Sigma_{\alpha=1}^{d} M_{l, \alpha ; n(l, \alpha)=0 ;+} \times \\
& \left(\Sigma_{l^{\prime}=1}^{N} \Sigma_{\alpha^{\prime}=1}^{d}[D[[n=1] ; \epsilon]]_{l, \alpha ; l^{\prime}, \alpha^{\prime}} M_{l^{\prime}, \alpha^{\prime} ; n\left(l^{\prime}, \alpha^{\prime}\right)=1 ;-}\right) g([n=0])
\end{aligned}
$$

The (continued fraction) operator $D[[n=1] ; \epsilon]$ is Hermitian with non-negative eigenvalues and has, as a square matrix, the matrix elements $[D[[n=1] ; \epsilon]]_{l, \alpha ; l^{\prime}, \alpha^{\prime}}$ (possibly, with $\epsilon \rightarrow 0$ ). The justification generalizes that for $D[1 ; \epsilon]$ in Section 2.2; see $[15,16,20]$. The initial condition is $W_{c, e q}([0])^{-1 / 2} W_{c, i n}([0])$. Equation (102) describes the Brownian-like motion of $\mathrm{N}$ random walkers: the interaction of each walker with the other $N-1$ ones is contained in $V$.

The structure of Equation (31), with $V_{i, j} \neq 0$ between any pair of particles and $D[[n=1] ; \epsilon]$ replaced by a constant, is similar to that of the linear Smoluchowski equation in the standard Rouse model for (linear) polymer dynamics [25], except for the following difference. For the polymer, one deals with a linear three-dimensional chain of many atoms, and the interaction is $\neq 0$ only (or dominant) between successive pairs of neighboring atoms along the chain (each of which performs Brownian-like motion). Another physical motivation of our approach would be to provide irreversible evolution equations for the actual (real gas) system, which, being different from those in the standard non-equilibrium BBGKY hierarchy, would have, in the simplest case (Equation (31)), a structure mimicking the one in the standard Rouse model for polymer dynamics, namely, resembling Brownian motion for $N$ interacting random walkers, and would allow for generalizations (by using $g([n])$, with $n>0$ ).

Let $d=3$. We shall introduce the $t$-dependent Lyapunov function:

$$
L=\frac{1}{2} \int \prod_{i=1}^{N} \prod_{\alpha=1}^{3} d x_{i, \alpha} g([n=0])^{2}
$$

upon integrating in $-\infty<x_{i, \alpha}<+\infty$ for any $i, \alpha$. Like for Equation (15) in Section 2.2, Equation (31) implies: 
Then, the actual $L$ is a non-decreasing function in the time evolution. The generalization of a Lyapunov function for $n_{0}>1$, also fulfilling the generalization of Equation (33), can be made by extending [15,17] and will be omitted here. Let the classical many-particle system be at thermal equilibrium at $T$. Then:

$$
L=\frac{1}{2} \int \prod_{i=1}^{N} \prod_{\alpha=1}^{3} d x_{i, \alpha} q_{0}^{3 N} \pi^{3 N / 4} \exp [-\beta V] \equiv L_{e q}
$$

As is well known, in equilibrium statistical mechanics, the equilibrium entropy, $S_{\text {eq }}$, is defined, up to a constant $S_{e q, 0}$, by:

$$
\exp \left[\frac{S_{e q}-S_{e q, 0}}{k_{B}}\right]=\int\left[\prod_{i=1}^{N} \prod_{\alpha=1}^{3} d x_{i, \alpha}\right] \int[d q] W_{c, e q}
$$

Upon comparing Equations (34) and (35), we get:

$$
\exp \left[\frac{S_{e q}-S_{e q, 0}}{k_{B}}\right]=2 \pi^{3 N / 4} L_{e q}
$$

In our statistical mechanics approach, the situation seems somewhat paradoxical. In fact, let us start out from the $t$-independent structure in Equation (36) in order to go beyond equilibrium by letting $t$ vary. That is, one would try tentatively a non-equilibrium entropy $S(t)$, such that $\exp \left[\frac{S(t)}{k_{B}}\right]$ would be proportional to $L(t)$. However, in so doing, one faces a paradox: as $L(t)$ decreases as $t$ increases, such an $S(t)$ would also be forced to decrease, which strongly contradicts, in principle, what one expects from any physically acceptable non-equilibrium entropy. Then, if a $t$-dependent non-equilibrium entropy exists, it should not be related to $L(t)$ through some structure like "exp $\left[\frac{S(t)}{k_{B}}\right]$ proportional to $L(t)$ ". Alternatively, we recall that, at thermal equilibrium, one introduces the information, $I_{e q}$, as $-S_{e q}$, and that if a non-equilibrium information, $I(t)$, could be defined in general, one would expect that it would decrease as $t$ increases. Then, an attempt to introduce such a non-equilibrium information, $I(t)$, through a structure like " $\exp \left[\frac{-I(t)}{k_{B}}\right]$ proportional to $L(t)$ ", would not work either; as such, an $I(t)$ would be an increasing function.

There has been much interesting work aimed at extending, in different frameworks (thermodynamical, statistical, etc.), the definition of a $t$-dependent entropy, $S(t)$, to non-equilibrium phenomena, in such a way that the inequality $d S(t) / d t \geq 0$ would hold. Rate equation dynamics following the path of steepest entropy ascent constitute an interesting research subject [28]. At present, several different non-equilibrium entropies have been proposed and do characterize, in general settings, the notion of evolution [29-32] (to the best of the present author's knowledge). So far, neither a general agreement seems to have been reached on a unique non-equilibrium entropy $S(t)$ valid for different frameworks [29,32], nor an accepted definition of it on the basis of non-equilibrium statistical mechanics appears to exist (to the best of the present author's knowledge). Further perspectives are given by other interesting presentations, like [33].

Both in case that a general definition of a unique non-equilibrium entropy does not exist or, in the case that it could be formulated at the end, our proposal for a partial way out towards the characterization of, at least, an approximate arrow of time in the actual moment approach to non-equilibrium classical statistical mechanics is the following. Once the long-time approximation has been carried out as indicated above, we have studied a $t$-dependent function, $L$, through Equation (32), the variation of which seems to 
be adequate to define an arrow of time, although it does fail to provide a non-equilibrium entropy!A previous study of $L$ was limited to the framework of classical Brownian motion [25] and did not relate it to either non-equilibrium classical statistical mechanics for $N$ particles or (to our knowledge) to any sort of non-equilibrium entropy. Our proposal could possibly complement partially different proposals by other authors characterizing evolution; see [1-4,9,29-34].

\section{Open Quantum-Mechanical One-Dimensional System without Dissipation: General Aspects}

\subsection{Some General Aspects}

We shall consider a quantum Brownian particle $(q B p)$ of mass $m(>0)$ and momentum operator $-i \hbar(\partial / \partial x)$, in one spatial dimension, $x$, with (Hermitian) quantum Hamiltonian:

$$
H=-\frac{\hbar^{2}}{2 m} \frac{\partial^{2}}{\partial x^{2}}+V
$$

with a real potential $V=V(x)$ ( $\hbar$ being Planck's constant). We shall suppose that $V(x) \geq 0$ and, having in mind possible generalizations to many degrees of freedom, that $V(x)=V(-x)$ (parity being a constant of motion). We shall also suppose that $V(x)$ and all $d^{n} V(x) / d x^{n}, n=1,2,3 \ldots$, are continuous for any $x$; a study for $V$ 's with finite discontinuities will be reported later (see, in particular, Appendices $\mathrm{C}$ and D). If $\varphi_{j}(x)$ is a suitably normalized eigenfunction of $H: H \varphi_{j}(x)=E_{j} \varphi_{j}(x), j$ being a suitable label. We shall deal with two classes of potentials, $V(x)$, depending on their behavior as $|x| \rightarrow+\infty$, and characterized as follows. The first class of $V(x)$ 's fulfills $V(x) \rightarrow 0$ quickly, as $|x| \rightarrow+\infty$. Then, all eigenvalues, $E_{j}$, of $H$ sweep the continuous positive real half-line: $0 \leq E_{j}<+\infty$. Such a purely continuous spectrum has, typically, a double degeneracy, associated with two different asymptotic conditions (incoming plane waves) at $x \rightarrow \pm \infty$, with the same energy, $E_{j}$. Then, $j$ is a continuous variable $(-\infty<j<+\infty)$, which labels all states and distinguishes degenerate ones (see Appendices $\mathrm{D}$ and G). The second class of $V(x)$ 's fulfill as $|x| \rightarrow+\infty, V(x) \rightarrow+\infty$ (proportional to $x^{2 n}$, for positive integer $n$ ), and they correspond specifically to harmonic/anharmonic oscillators (polynomials; see Section 5.1, where the spectra of the corresponding Hamiltonians is discussed). All items in this Section will apply to both classes of $V(x)$ 's, unless otherwise stated.

For general aspects on quantum open systems, see [34-38] and the references therein. We consider the non-equilibrium statistical evolution of a $q B p$ subject to $V(x)$ and in the presence of a $h b$ at thermal equilibrium at $T$, with negligible external dissipation, due to the $h b$. The time evolution for $t>0$ of the $q B p$ is given by the density operator $\rho=\rho(t)$ (a statistical mixture of quantum states), with the initial condition $\rho(t=0)=\rho_{i n} . \rho(t)$ for $t>0$ and $\rho_{i n}$ are Hermitian and positive-definite linear operators acting in the Hilbert space spanned by the set of all eigenfunctions, $\varphi_{j}(x)$, of $H$. Unless otherwise stated, we shall not impose that $\rho(t)$ be normalized. The time evolution of the $q B p$ is described by the operator equation $([H, \rho]=H \rho-\rho H)$ :

$$
\frac{\partial \rho}{\partial t}=\frac{1}{i \hbar}[H, \rho]
$$


We consider the matrix element, $\langle x-y|\rho(t)| x+y\rangle$, of $\rho(t)$ in generic eigenstates, $|x-y\rangle,|x+y\rangle$, of the quantum position operator. The quantum Wigner function $W=W(x, q ; t)$, determined by $\rho$, is [6,7]:

$$
W(x, q ; t)=\frac{1}{\pi \hbar} \int_{-\infty}^{+\infty} d y \exp \left[\frac{i 2 q y}{\hbar}\right]\langle x-y|\rho(t)| x+y\rangle
$$

The initial non-equilibrium Wigner function at $t=0$ is $W_{i n}$, given by Equation (39) if $\rho=\rho_{i n}$. For $t>0$, the exact dissipationless quantum master equation for $W[6,7]$ is:

$$
\begin{aligned}
\frac{\partial W(x, q ; t)}{\partial t}= & -\frac{q}{m} \frac{\partial W(x, q ; t)}{\partial x}+M_{Q} W \\
M_{Q} W= & \int_{-\infty}^{+\infty} d q^{\prime} W\left(x, q^{\prime} ; t\right) \int_{-\infty}^{+\infty} \frac{i d y}{\pi \hbar^{2}}[V(x+y)-V(x-y)] \\
& \times \exp \left[\frac{i 2\left(q-q^{\prime}\right) y}{\hbar}\right]=\frac{d V}{d x} \frac{\partial W}{\partial q}-\frac{\hbar^{2}}{3 ! 2^{2}} \frac{d^{3} V}{d x^{3}} \frac{\partial^{3} W}{\partial q^{3}}+\cdots
\end{aligned}
$$

As $\hbar \rightarrow 0$, Wigner's Equation (40) becomes, formally, by dropping all $\hbar$-dependent terms (containing $\partial^{n} W / \partial q^{n}, n=3,5, \ldots$ ) in Equation (41), the classical Liouville Equation (1), with $W \rightarrow W_{c}$ [6,7]. All terms in the series in Equation (41) contribute, in general, to $V$ 's in the first class. For $V$ 's in the second class, there is some $n_{1}$, such that $d^{n} V / d x^{n} \equiv 0$ for $n>n_{1}$. We shall suppose that, as $|q| \rightarrow+\infty$, $W(x, q ; t) \rightarrow 0$ quickly, for fixed $x$ and $t$, so that $\int_{-\infty}^{+\infty} d q W(x, q ; t) q^{n}$ converges, for any integer $n \geq 0$. We shall check the validity of such an assumption in specific cases in Section 4.4 and Appendix C. Under the latter assumption, Equation (40) readily implies that $(\partial / \partial t) \int_{-\infty}^{+\infty} d x \int_{-\infty}^{+\infty} d q W(x, q ; t)=0$.

A stationary density operator, $\rho_{s t}$, for the system fulfills Equation (38), with $\partial \rho / \partial t=0$. Then, $\rho_{s t}=f(H)$ is a function of $H$ only (in the actual one-dimensional system, the constants of motion being $H$ and parity). Then, $\rho_{s t}$ has the matrix elements:

$$
\left\langle x-y\left|\rho_{s t}\right| x+y\right\rangle=\sum_{j} f\left(E_{j}\right) \varphi_{j}(x-y) \varphi_{j}(x+y)^{*}
$$

For a continuous spectrum, $\sum_{j}$ is a short-hand notation denoting integration over the whole continuous spectrum of $j\left(\int_{-\infty}^{+\infty} d j\right)$, with $\sum_{j} \varphi_{j}(x) \varphi_{j}(y)^{*}=\delta(x-y), \delta$ denoting the Dirac delta function. For a purely discrete spectrum, $\sum_{j}$ stands for the infinite sum over the denumerably infinite set of all $E_{j}$ 's: see Section 5.1. $f\left(E_{j}\right)$ are real and non-negative constants, for all $E_{j}$. In turn, $\rho_{s t}$ determines the stationary Wigner function, $W_{s t}(x, p)$, through Equation (39). Then, $W_{s t}$ fulfills:

$$
-\frac{q}{m} \frac{\partial W_{s t}}{\partial x}+M_{Q} W_{s t}=0, \frac{\partial W_{s t}}{\partial t}=0
$$

We shall also suppose that $W_{s t} \rightarrow 0$ quickly for $|q| \rightarrow+\infty$. The general structure of $\rho_{i n}$ is:

$$
\left\langle x-y\left|\rho_{i n}\right| x+y\right\rangle=\sum_{j, j^{\prime}} c_{j, j^{\prime}} \varphi_{j}(x-y) \varphi_{j^{\prime}}(x+y)^{*}
$$

where $c_{j, j^{\prime}}$ define a Hermitian non-negative matrix. In general, $\left[H, \rho_{i n}\right] \neq 0$. See [38] for theorems and constructive methods to find stationary solutions of Equation (43).

There exist two exact integral relationships for any $W$ fulfilling Equation (40), for any $V, x$ and $t$ :

$$
\begin{aligned}
& \frac{\partial\left[\int d q W(x, q ; t)^{2}\right]}{\partial t}=-\frac{1}{m} \frac{\partial\left[\int d q q W(x, q ; t)^{2}\right]}{\partial x} \\
& \frac{\partial\left[\int d q W_{s t}(x, q) W(x, q ; t)\right]}{\partial t}=--\frac{1}{m} \frac{\partial\left[\int d q q W_{s t}(x, q) W(x, q ; t)\right]}{\partial x}
\end{aligned}
$$


the integrations over $q$ being performed in $-\infty<q<+\infty$. $W_{s t}$ is an arbitrary stationary Wigner function. Equation (45) is proven by multiplying Equation (40) by $W$, integrating over $q$, performing partial integrations and recalling that as $|q| \rightarrow+\infty, W(x, q ; t) \rightarrow 0$ quickly. Equation (46) is derived by multiplying Equation (40) by $W_{s t}$, operating similarly and using the first Equation (43). Equations (45) and (46) are new, to the best of the present author's knowledge.

The main part of this work will generalize, wherever possible, the developments in Section 2.2 to the (much more difficult) Wigner Equation (40).

\section{2. $W$ and $W_{\text {st }}$ As Quasi-Definite Functionals in Momentum}

We now remind ourselves of the known quantum difficulty: neither $W$ nor $W_{s t}$ can be warranted to be $\geq 0$ (negativity), in general [6,7]. As stated in [39], a necessary and sufficient condition for the Wigner function associated with a Schrodinger wave function being $\geq 0$ is that the latter is the exponential of a quadratic polynomial [39]. See also [40,41]. As a nontrivial illustration, the Wigner functions, $W$, associated with several eigenstates of the Morse potential, have been studied by combining analytical and numerical methods: negative values of the $W$ associated with the ground state are reported in $[42,43]$. The latter two references and [44] present negative values of the $W$ 's associated with some excited states. Even if $W_{s t}<0$, the domain in which that occurs cannot be large and has to be consistent with the fact that both $\int_{-\infty}^{+\infty} d x W_{s t}$ and $\int_{-\infty}^{+\infty} d q W_{s t}$ are $\geq 0$.

In order to be able to proceed in spite of $W<0$, we shall now invoke, in an outline, an acceptable mathematical framework based upon the theory of orthogonal polynomials [26]. Let us consider a kernel $K=K(y)$ (which could be $\leq 0$ ), a set of functions $f=f(y)$ and the following functional, $L_{K}$, determined by the kernel, $K: f \rightarrow L_{K}[f]=\int_{-\infty}^{+\infty} d y K(y) f(y)$. We shall suppose that all integrals over $y$ are convergent. Let us consider, successively: $\mu_{n}=L_{K}\left[y^{n}\right], n=0,1,2,3 \ldots$, the set of all $(S+1) \times(S+1)$ matrices $M_{S}(S=0,1,2,3, \ldots)$ with the $(i, j)$-th element equal to $\mu_{i+j}(i, j=0, \ldots, S)$ and their determinants, $\operatorname{Det}\left[M_{S}\right]$. By definition, the functional, $L_{K}$, is quasi-definite if $\operatorname{Det}\left[M_{S}\right] \neq 0$ for any $S=0,1,2,3, \ldots$ [26]. If $L_{K}$ is a quasi-definite functional, then a theorem [26] implies: (i) the existence of a family of orthogonal polynomials, named here as $H_{Q, n}=H_{Q, n}(y)$, with weight function $K$ (even if $K<0$ in some domain in $y$ ); and (ii) that the polynomials, $H_{Q, n}$, fulfill a three-term recurrence relation analogous to Equation (21), in which the ( $y$-independent) counterparts of the $C_{c, n}$ 's are not warranted to be $>0$.

Let $q_{0}$ be some fixed ( $x$-independent) momentum and let us replace $q$ by $y=q / q_{0}$. We shall suppose in all that follows that, regarding their $y$-dependences, the Wigner function, $W$, and any $W_{s t}$ determine, respectively, quasi-definite functionals, $L_{W}$ (for any $x$ and $t$ ) and $L_{W_{s t}}$ (for any $x$ ). The interest of the assumption is obvious: if it holds (as we suppose), it implies the existence of orthogonal polynomials. To check that assumption for any $W$ and $W_{s t}$, in general, lies outside our scope: its validity for $L_{W_{s t}}$ will be checked (and confirmed) in one interesting case in Section 5.2 and in Appendix E.

\subsection{Equilibrium Wigner Function}

We shall now consider the equilibrium Wigner function $W_{s t}=W_{e q}$, fulfilling Equation (43) and accounting for thermal equilibrium at $T$ of the $q B p$ with the $h b$. Like in the classical case, the solutions 
of Equations (40) and (41) are not expected to approach $W_{e q}$ exactly, unless some approximation is made. $W_{e q}$ arises from the canonical ( $t$-independent) density operator $\rho_{e q}=\exp [-\beta H]$. Then:

$$
\left\langle x-y\left|\rho_{e q}\right| x+y\right\rangle=\sum_{j} \exp \left[-\beta E_{j}\right] \varphi_{j}(x-y) \varphi_{j}(x+y)^{*}
$$

$\sum_{j}$ in Equation (47) has the same meaning as in Equation (42). $\rho_{e q}$ determines $W_{e q}(x, q)$, through Equation (39). $W_{e q}(x, q)$ is neither Gaussian in $q$ nor known in closed form in general [6,7], and the dependences on $q$ and $x$ do not factorize. $W_{e q}(x, q)$ for a repulsive finite square well is given in Appendix D.

It is easy to show that the imaginary part, $\operatorname{Im} W_{e q}(x, q)$, of $W_{e q}(x, q)$ vanishes for any $x$ and $q$, that is, $W_{e q}(x, q)$ is real. Let $E_{j}$ sweep the continuous positive real half-line: $0 \leq E_{j}<+\infty$ ( $V$ in first class). Let $\beta$ become complex $(\beta=\operatorname{Re} \beta+i \operatorname{Im} \beta$ ) and wander in the right-half-plane $\operatorname{Re} \beta>0$. Then, one sees that $W_{e q}(x, q)$ is an analytic function of $\beta$ in the right-half-plane $\operatorname{Re} \beta>0$, for fixed $x$ and $q$.

\subsection{Orthogonal Polynomials $H_{Q, n}$ Generated by $W_{s t}$ and by $W_{e q}$}

We shall introduce the (unnormalized) polynomials in $y\left(=q / q_{0}\right), H_{Q, n}=H_{Q, n}(y)$ $(n=0,1,2,3, \ldots)$, orthogonalized in $y$ (for fixed $x$ ) by using a generic stationary $W_{s t}$ (Equation (43)) as the weight function. By choosing $H_{Q, 0}(q)=1$, for $n \neq n^{\prime}$ and any $x$ (left unintegrated), we impose:

$$
\int_{-\infty}^{+\infty} d y W_{s t} H_{Q, n}(y) H_{Q, n^{\prime}}(y)=0
$$

The $H_{Q, n}$ 's, depending parametrically on $x$ for $n \geq 1$, will be used for the time evolution. For $V$ 's belonging to the first class, we shall look for the $H_{Q, n}(y)$ 's as:

$$
H_{Q, n}(y)=y^{n}+\sum_{j=1}^{n} \epsilon_{Q, n, n-j} y^{n-j}
$$

$\epsilon_{Q, n, n-j}$ being $y$-independent (but $x$-dependent, in general). One has $\epsilon_{Q, n, n-j}=0$ for odd $j$, so that $H_{Q, n}(-y)=(-1)^{n} H_{Q, n}(y)$. The $\epsilon_{Q, n, n-j}$ 's are given by equations entirely similar to those yielding the $\epsilon_{c, n, n-j}$ 's in Appendix A (Equations (73)-(76)), provided that the quantum $<y^{n}>$ be now understood as $\int_{-\infty}^{+\infty} d y W_{s t} y^{n} / \int_{-\infty}^{+\infty} d y W_{s t}$. The orthonormalized polynomials are $H_{Q, n}(q) /\left(h_{Q, n}\right)^{1 / 2}$, with an (x-dependent) normalization factor, $h_{Q, n}$, defined through Equation (3), by replacing $W_{c, s t}$ by $W_{s t}$ and $H_{c, n}$ by $H_{Q, n}$. One could also express the same $H_{Q, n}$ 's as suitable sums of Hermite polynomials: such an alternative, although consistent, will not be followed for $V$ 's in the first class (but it will in Section 5.1, for $V$ 's in the second class). The above definitions and statements also hold, in particular, for the polynomials $H_{Q, e q, n}=H_{Q, e q, n}(y)$ determined by $W_{s t}=W_{e q}$, with suitable choices of $q_{0}$ (to be discussed later). For another discussion of the $H_{Q, e q, n}$ 's, see [20].

We shall not focus on cases in which $V(x) \rightarrow 0$ quickly for $|x| \rightarrow+\infty$ and can be $\leq 0$ (attractive), except for the following comments (and a short one in Section 5.1). In such a case, $H$ has a general spectrum; both discrete (finite number of bound states) plus continuous spectra. We shall justify briefly the motivation for such an exclusion. Let us consider for a short while the attractive $\delta$-function potential $V(x)=-V_{0} \delta(x), V_{0}$ being a positive constant. Then, $H$ has a unique bound state, namely, 
$\varphi_{0}(x)=A \exp [-\alpha|x|]$, with $\alpha=m V_{0} / \hbar^{2}>0, A=\alpha^{1 / 2}$. A direct computation of $W_{e q}$ at $x=0$ in Equation (39), by using Equation (47) and keeping only the contribution of $\varphi_{0}(x)$ (say, at very low $T$ ), shows that such a contribution to $W_{e q}(x=0, q)$ equals a constant times $\left[\alpha^{2}+(q / \hbar)^{2}\right]^{-1}$. The latter contribution to $W_{e q}(x=0, q)$ has a slow decrease as $q$ increases, and hence, it does not allow one to define orthogonal polynomials, $H_{Q, e q, n}(y)$, for $n \geq 2$, because one finds divergent integrals in $q$. A similar study for $V(x)$ corresponding to an attractive finite square well having, for simplicity, only one bound state yields a contribution to $W_{e q}(x=0, q)$ having another slow decrease as $q$, which also prevents the construction of an infinite family of $H_{Q, e q, n}(y)$ 's.

\section{Denumerably Infinite Purely Discrete Spectrum}

\subsection{Some General Aspects}

Here, we treat $V$ 's in the second class (polynomials: harmonic/anharmonic oscillators). Then, $H$ is defined in a denumerably infinite Hilbert space, and it has a nondegenerate denumerably infinite discrete spectrum, $E_{j}, j=1, \ldots$ ( $E_{j} \geq 0$, without loss of generality), the continuous spectrum being absent. Let $\varphi_{0}$ be the ground state with energy $E_{0}$, and let the successive nondegenerate eigenvalues be ordered as $j=1,2,3 \ldots$, with $E_{0}<E_{1}<E_{2}<E_{3}<\ldots$. All eigenfunctions, $\varphi_{j}$, are square-integrable and, by assumption, normalized. As $V(x)=V(-x)$, one has $: \varphi_{j}(-y)=(-1)^{j} \varphi_{j}(y)$. Then, Equations (47) and (39), with $f\left(E_{j}\right)=\exp \left(-\beta E_{j}\right)$ give:

$$
\begin{aligned}
& W_{e q}(x=0, q=0)=\frac{1}{\pi \hbar} \int_{-\infty}^{+\infty} d y \sum_{j} \exp \left[-\beta E_{j}\right] \varphi_{j}(-y) \varphi_{j}(y)^{*}= \\
& \frac{1}{\pi \hbar}\left[\left(\exp \left[-\beta E_{0}\right]-\exp \left[-\beta E_{1}\right]\right)+\left(\exp \left[-\beta E_{2}\right]-\exp \left[-\beta E_{3}\right]\right)+\ldots .\right]
\end{aligned}
$$

It follows that $W_{e q}(x=0, q=0)>0$ for any $T$. The same argument indicates that, for sufficiently low $T$ and adequately small $|x|$ and $|q|, W_{e q}(x, q) \simeq(\pi \hbar)^{-1} \exp \left[-\beta E_{0}\right]$, which is non-negative: that is, $W_{e q}(x, q)$ is dominated by the ground state $\varphi_{0}$ with energy $E_{0}$. This dominance of $\varphi_{0}$ for small $T, x$ and $q$ formally holds for $V$ 's having a general (discrete finite plus continuous) spectrum.

Let $T=0$ strictly. It follows that: (i) $W_{e q}$ is proportional to $(\pi \hbar)^{-1} \int_{-\infty}^{+\infty} d y \exp \left[\frac{i 2 q y}{\hbar}\right] \varphi_{0}(x-y) \varphi_{0}(x+$ $y)^{*}$ (we have factored out $\exp \left[-\beta E_{0}\right]($ at $T \rightarrow 0$ ), which leads us to discard any other contribution different from the ground state one to $W_{e q}$ in the $T \rightarrow 0$ limit); (ii) any other $W_{s t}$ is necessarily proportional to $W_{e q}$; (iii) the initial condition, $W_{i n}$, is proportional to $W_{e q}$; and (iv) $W$ is also proportional to $W_{e q}$ and, hence, it is equal to $W_{i n}$ and $t$-independent. Cases with low $T$ will be treated later.

For $V$ 's in the second class, we choose another $q_{0}^{\prime}$ ( $\neq$ the $q_{0}$ employed in Equation (49), in principle) and, instead of using Equation (49), the same orthogonal polynomials, $H_{Q, n}(y)(n=0,1,2,3, \ldots)$, will be searched for being equal to the standard Hermite polynomial, $H_{n}(y)$, plus another polynomial in $y$ of a degree smaller than $n$. Then:

$$
H_{Q, n}(y)=H_{n}(y)+\sum_{j=1}^{n} \sigma_{n, n-j} H_{n-j}(y)
$$


with $n=1,2,3, \ldots$ and ( $y$-independent, but $x$-dependent) coefficients $\sigma_{n, n-j}$. One has: $H_{Q, n}(y)=H_{n}(y)$, for $n=0,1$ and $\sigma_{n, n-j}=0$ for odd $j$. For generic $q_{0}$ and $q_{0}^{\prime}$ (unrelated to each other), the $H_{Q, n}(y)$ 's in Equation (51) are equal to those in Equation (49) times $\left(2 q_{0} / q_{0}^{\prime}\right)^{n}$, which, in general, is $\neq 1$.

\subsection{One-Dimensional Quantum Harmonic Oscillator}

We shall treat a quantum harmonic oscillator, with frequency $\omega(>0)$ and $V=V(x)=m \omega^{2} x^{2} / 2$. Let the quantum oscillator be at thermal equilibrium at $T$ with some $h b$. The corresponding $\left\langle x-y\left|\rho_{e q}\right| x+y\right\rangle$ in Equation (47) is well known [45], and $W_{e q}$ reads:

$$
\begin{aligned}
W_{e q}(x, q)= & {\left[\frac{1}{2(\pi \hbar)^{2}(1+\cosh (\beta \hbar \omega))}\right]^{1 / 2} \exp \left[-\frac{m \omega(\cosh (\beta \hbar \omega)-1) x^{2}}{\hbar \sinh (\beta \hbar \omega)}\right] \times } \\
& \exp \left[-\frac{\sinh (\beta \hbar \omega) q^{2}}{m \hbar \omega(1+\cosh (\beta \hbar \omega))}\right]
\end{aligned}
$$

See [20] for the orthogonal polynomials for Equation (52). For simplicity, we shall set the ground state energy equal to zero and choose $\hbar \omega=1$ and $m=1$. For the harmonic oscillator $V(x)$, the series expansion of the operator, $M_{Q}$, into powers of $\hbar^{2}$ reduces exactly to the first term shown in Equation (41). Then, Equations (40) and (41) reduce to a quantum equation formally similar to the classical Equation (1):

$$
\frac{\partial W}{\partial t}+q \frac{\partial W}{\partial x}-x \frac{\partial W}{\partial q}=0
$$

First, let the quantum oscillator be in the ground state. The stationary Wigner function is:

$$
W_{s t}(x, q)=\frac{2}{\pi} \exp \left[-2\left(x^{2}+q^{2}\right)\right]>0
$$

The orthogonal polynomials for Equation (52) yield, as $\beta \rightarrow 0$, those for Equation (54).

Next, let the quantum oscillator be in the first excited state and let $y=2^{1 / 2} q$ in the present case. The associated stationary Wigner function, of considerable interest in quantum optics, is [46]:

$$
\begin{aligned}
W_{s t}(x, q) & =\frac{2}{\pi}\left[4\left(x^{2}+q^{2}\right)-1\right] \exp \left[-2\left(x^{2}+q^{2}\right)\right] \\
& =\frac{2}{\pi}\left[\frac{1}{2} H_{2}(y)+4 x^{2}\right] \exp \left[-\left(2 x^{2}+y^{2}\right)\right]
\end{aligned}
$$

$H_{2}(y)$ being the standard Hermite polynomial. One easily confirms that Equation (55) is a stationary solution of Equation (53)). $W_{s t}(x, q)$ is negative in the finite domain in which $4\left(x^{2}+q^{2}\right)<1$ [46]. This case provides an interesting example, enabling us to justify that $W_{s t}(x, q)$ in Equation (55) is indeed a quasi-definite functional regarding the $y$ (or $q$ ) dependence for any $x$ (except at a set of zero measure): see Appendix E. The polynomials, $H_{Q, n}(y)(n=0,1,2,3, \ldots)$, orthogonalized in $y$ (for fixed $x$ ) by using $W_{s t}$ in Equation (55) as the weight function, are given in Equation (51); the low-order coefficients, $\sigma_{n, n-j}$, are given in Appendix E.

$W_{e q}$, given in Equation (52), is positive, and one could ask how the negativity of Equation (55) can be compensated for. To answer that question, we shall suppose that $T$ is so low that the actual $\sum_{j}$ reduces 
just to the contributions of the ground state and of the first excited one. Then, the corresponding Wigner function is:

$$
W_{e q}(x, q) \simeq \frac{2}{\pi} \exp \left[-2\left(x^{2}+q^{2}\right)\right]\left[1+\exp \left[-\beta E_{1}\right]\left(4\left(x^{2}+q^{2}\right)-1\right)\right]
$$

$E_{1}>0$ being the energy of the first excited state. Clearly, $W_{e q}(x, q)>0$ for any $x$ and $q$, which is consistent with the positivity of Equation (52).

\section{Non-Equilibrium Hierarchy}

\subsection{V's in the First Class}

We consider only $V$ 's in the first class (all its derivatives exist and are continuous) and suppose that $T$ is neither high nor low. We shall analyze general off-equilibrium situations by using Equations (40) and (41). We consider a generic $W_{s t}$. The $H_{Q, n}(y)$ 's in Equation (49) lead to the new moments $(n=0,1,2, \ldots)$ :

$$
W_{n}=W_{n}(x ; t)=\int_{-\infty}^{+\infty} d y H_{Q, n}(y) W
$$

The initial condition, $W_{i n, n}$, for $W_{n}$ is obtained by replacing $W$ by $W_{i n}$ in Equation (56). One has the following (formal) expansion for $W$, which generalizes Equation (5):

$$
W=W_{s t}(x, q) \sum_{n=0}^{+\infty} W_{n}(x ; t) \frac{H_{Q, n}(y)}{h_{Q, n}}
$$

For $W=W_{s t}(x, q)$, Equation (56) yields $W_{s t, n}=0$ if $n>0$, and $W_{s t, 0}=h_{Q, 0}$. The transformation of Equations (40) and (41) into a linear hierarchy for the new moments, $W_{n}$, for a general $W_{s t}$, can be carried out as in the classical case. Through additional computations and cancellations, we have obtained the first five equations in that quantum hierarchy.

$$
\begin{aligned}
\frac{\partial W_{0}}{\partial t}= & -\frac{q_{0}}{m} \frac{\partial W_{1}}{\partial x} \\
\frac{\partial W_{1}}{\partial t}= & -\frac{q_{0}}{m} \frac{\partial W_{2}}{\partial x}+\frac{q_{0}}{m} \frac{\partial}{\partial x}\left[\left(\epsilon_{Q, 2,0}\right) W_{0}\right]-\frac{1}{q_{0}} \frac{\partial V}{\partial x} W_{0} \\
\frac{\partial W_{2}}{\partial t}= & -\frac{q_{0}}{m} \frac{\partial W_{3}}{\partial x}+\frac{q_{0}}{m} \frac{\partial}{\partial x}\left[\left(\epsilon_{Q, 3,1}-\epsilon_{Q, 2,0}\right) W_{1}\right]+\frac{q_{0}}{m} \frac{\partial \epsilon_{Q, 2,0}}{\partial x} W_{1}-\frac{2}{q_{0}} \frac{\partial V}{\partial x} W_{1} \\
\frac{\partial W_{3}}{\partial t}= & -\frac{q_{0}}{m} \frac{\partial W_{4}}{\partial x}+\frac{q_{0}}{m} \frac{\partial}{\partial x}\left[\left(\epsilon_{Q, 4,2}-\epsilon_{Q, 3,1}\right) W_{2}\right]+\frac{q_{0}}{m} \frac{\partial \epsilon_{Q, 3,1}}{\partial x} W_{2}-\frac{3}{q_{0}} \frac{\partial V}{\partial x} W_{2} \\
\frac{\partial W_{4}}{\partial t}= & -\frac{q_{0}}{m} \frac{\partial W_{5}}{\partial x}+\frac{q_{0}}{m} \frac{\partial}{\partial x}\left[\left(\epsilon_{Q, 5,3}-\epsilon_{Q, 4,2}\right) W_{3}\right]+\frac{q_{0}}{m} \frac{\partial \epsilon_{Q, 4,2}}{\partial x} W_{3}-\frac{4}{q_{0}} \frac{\partial V}{\partial x} W_{3}+ \\
& +\frac{\hbar^{2}}{2^{2} q_{0}^{3}} \frac{\partial^{3} V}{\partial x^{3}}\left[-6+\frac{\epsilon_{Q, 4,2}}{\epsilon_{Q, 2,0}}\right] W_{1}
\end{aligned}
$$

The first four Equations (58)-(61) are formally identical, respectively, to those in the classical case (Equations (22)-(25)), provided that $W_{c, n}$ 's are replaced by $W_{n}$ 's and $\epsilon_{c, n, n-j}$ 's by $\epsilon_{Q, n, n-j}$ 's, for $n=0,1,2,3$. On the other hand, for a general $W_{s t}$, Equation (62) (for $n=4$ ) acquires a new term 
(of quantum origin) containing $W_{1}$, and so, it differs from its classical counterpart, Equation (26). The reason for the similarities for $n=0,1,2,3$ and for the difference if $n=4$ is that the quantum corrections in Equations (40) and (41) manifest themselves only at order $\hbar^{2}$ and then, in turn, in the equations in the hierarchy at orders $n \geq 4$. The computation and cancellations leading to Equation (62) are very lengthy and painful, and we shall omit them. We have confirmed that $W_{0}=h_{Q, 0}$ and $W_{n}=0$ for $n=1,2,3,4,5$ solve the hierarchy Equations (58)-(62), with $\partial W_{n} / \partial t=0$.

Let us now consider Equations (58)-(62) for the case $W_{s t}=W_{e q}$, describing thermal equilibrium with the $h b$. After further analysis, the new term containing $\hbar^{2} W_{1}$ in Equation (62) is not seen to disappear, but to be $\neq 0$ still, for generic $q_{0}$. For any $W_{s t}$ and $V \neq 0$, the equation for $\partial W_{n} / \partial t$ for any $n \geq 5$ contains a dependence on $W_{n+1}$, given below in Equation (64), but no other dependences on higher order moments. On the other hand, the fact that the full quantum equation for $n=4$ does contain a term of order $\hbar^{3}$ in $W_{1}$ implies that the quantum hierarchy is not a three-term one due to moments $W_{n^{\prime}}$ with $n^{\prime}<n$, neither for a generic $W_{s t}$ nor for $W_{e q}$. The most general non-equilibrium hierarchy is:

$$
\begin{aligned}
& \frac{\partial W_{n}}{\partial t}=-M_{n, n+1} W_{n+1}-\sum_{n^{\prime}=1}^{n} M_{n, n-n^{\prime}} W_{n^{\prime}} \\
& M_{n, n+1} W_{n+1} \equiv \frac{q_{0}}{m} \frac{\partial W_{n+1}}{\partial x}
\end{aligned}
$$

$M_{n, n^{\prime}=0}=0$ for any $n$, except for $n=1$ (with $n^{\prime}=0$ ). We emphasize that, in the non-equilibrium classical and quantum hierarchies (in Equations (22)-(26) for $W_{c, e q}$ and in Equations (63)-(64) for $W_{e q}$, respectively), the contributions from $W_{c, n+1}$ and from $W_{n+1}$ have always the same structures $\left(-\left(q_{0} / m\right) \partial W_{c, n+1} / \partial x\right.$ and $-\left(q_{0} / m\right) \partial W_{n+1} / \partial x$, with $n$-independent coefficients). On the other hand, the contributions from $W_{c, n-1}$ and from $W_{n^{\prime}}\left(0<n^{\prime} \leq n-1\right)$ do carry $n$-dependent coefficients, which increase with $n$. An example of such an $n$-dependence will be displayed, in the quasiclassical regime, by Equation (66). $\partial^{r} V / \partial x^{r}$ starts to contribute in Equation (63) for $n=r+1$.

\subsection{V's in the First Class: Reduction to Three-Term Hierarchy near the Classical Regime}

The comments after Equations (58)-(62) imply that, in the classical case $(\hbar \rightarrow 0)$, the quantum hierarchy reduces to the classical one, with $W_{s t} \rightarrow W_{c, s t}$.

We shall analyze the following more subtle simplification, only for $W_{s t}=W_{e q}$, with $q_{0}=(2 \mathrm{~m} / \beta)^{1 / 2}$ in the quasiclassical regime at high temperature, so that both $\beta$ and $\hbar(\neq 0)$ are small. $W_{e q}$, the orthogonal polynomials, $H_{Q, n}(y)$ 's, generated by it, the non-equilibrium solutions, $W$, the $W_{n}$ 's and the dynamical equations will be considered up to and including order $\hbar^{2}$ (higher orders in $\hbar$ being disregarded). We shall focus on whether the exact hierarchy Equations (58)-(62), which is not a three-term one, due to the contribution, $\hbar^{2} W_{1}$, in Equation (62), could become a three-term recurrence relation up to and including order $\hbar^{2}$. The analysis boils down to studying whether the coefficient multiplying $\hbar^{2} W_{1}$ in Equation (62), namely, $-6+\frac{\epsilon_{Q, 4,2}}{\epsilon_{Q, 2,0}}$, vanishes or not at the required order, which turns out to be order $\hbar^{0}$. In fact, as we are working to order $\hbar^{2}$ and there is an overall factor, $\hbar^{2}$, it will suffice to approximate $W_{e q}$ and $-6+\frac{\epsilon_{Q, 4,2}}{\epsilon_{Q, 2,0}}$ by $W_{c, e q}$ and $-6+\frac{\epsilon_{c, 4,2}}{\epsilon_{c, 2,0}}$, respectively. Thus, in order to evaluate $\epsilon_{c, 4,2}$ and $\epsilon_{c, 2,0}$ through Equations (73) and (74), we use $W_{c, e q}=\exp \left(-y^{2}\right) \exp [-\beta V]$. A direct computation shows that $-6+\frac{\epsilon_{c, 4,2}}{\epsilon_{c, 2,0}}=0$. Then, we see that up to and including order $\hbar^{2}$, Equations (58)-(62) become a quasiclassical three-term 
hierarchy. The simplification should hold for all higher values of $n=5,6,7, \ldots$ (say, for Equation (63)), in general. The last paragraph in Section 6.3 leads to the same conclusion, through a different procedure.

\subsection{V's in the Second Class: Examples of Quantum Hierarchies}

Here, we shall use the $H_{Q, n}(y)$ 's in Equation (51), which gives non-equilibrium moments equal to those in Equation (56) times $2^{2}\left(q_{0} / q_{0}^{\prime}\right)^{n+1}$ and the same formal hierarchy as in Section 6.1 (recast in terms of $\sigma_{n, n-j}$ 's). See [20] for the non-equilibrium three-term hierarchy for Equation (52). The off-equilibrium three-term hierarchy for the first excited state of the harmonic oscillator is outlined in Appendix E. Let us consider a quartic anharmonic oscillator: $V=V(x)=2^{-1} m \omega^{2} x^{2}+(4 !)^{-1} g x^{4}$, $g(>0)$ being a coupling constant. $W_{e q}$ and the associated orthogonal polynomials (for a suitable $q_{0}^{\prime}$ ) have been constructed in [20]. The resulting hierarchy for the non-equilibrium quantum moments, $W_{n}$ (for $W_{e q}$ ), has been given in [20] for $n=0,1,2,3$ and turns out to be a three-term one. However, further analysis indicates that the first quantum correction $\partial^{3} V / \partial x^{3}$ starts to contribute in equations in the hierarchy for $n \geq 4$, so that the whole non-equilibrium hierarchy is not a three-term one.

Just to illustrate the features met upon dealing with $n$-term hierarchies with $n>3$, we shall treat briefly the following four-term one, which is a simplification of the quantum ones:

$$
\frac{\partial W_{n}}{\partial t}=-M_{n, n+1} W_{n+1}-M_{n, n-1} W_{n-1}-M_{n, n-3} W_{n-3}
$$

where $M_{n, n+1} W_{n+1}$ could be either Equation (64) or $\left(q_{0}^{\prime} / 2 m\right)\left(\partial W_{n+1} / \partial x\right)$. The essential structure of the formal solution of the four-term hierarchy Equations (64) and (65) is outlined in Appendix F.

We shall now treat the same quasiclassical regime as in Section 6.2, also up to and including order $\hbar^{2}$, with $q_{0}^{\prime}=(2 m / \beta)^{1 / 2}$. In such a regime: (i) $W_{e q}$ is well known [6]; (ii) the $H_{Q, n}(y)$ 's have been constructed explicitly in terms of Hermite polynomials [21]; and (iii) it has been shown that the $W_{n}$ 's (determined by the latter $H_{Q, n}(y)$ ) fulfill the following three-term hierarchy for all values of $n, n=$ $0,1,2,3, \ldots .:\left(\partial W_{n} / \partial t\right)=-\left(q_{0}^{\prime} / 2 m\right)\left(\partial W_{n+1} / \partial x\right)-M_{n, n-1} W_{n-1}$ with the operators [21]:

$$
M_{n, n-1} W_{n-1}=\frac{2 n q_{0}^{\prime}}{m} \frac{\partial\left(1+4 a_{2}\right) W_{n-1}}{\partial x}+2\left[\frac{2 n}{q_{0}^{\prime}} \frac{\partial V}{\partial x}+\frac{2 q_{0}^{\prime} n(n-1)}{m} \frac{\partial a_{2}}{\partial x}\right] W_{n-1}
$$

and $a_{2}=\left(\beta^{2} \hbar^{2} / 48 m\right)\left(\partial^{2} V / \partial x^{2}\right)$.

The hierarchy Equation (66), with the $H_{Q, n}(y)$ 's in Equation (51), also applies in the quasiclassical regime for $V$ 's in the first class [21]. This is consistent with the simplification to a three-term hierarchy studied in Section 6.2.

\subsection{Long-Time Approximation in the Quantum Case: Pending Problems to Justify It}

First, we treat the four-term hierarchy of Equation (65) for $V$ 's in the second class and for $W_{e q}$, with the initial condition $W_{i n, 0} \neq 0, W_{i n, 0} \neq W_{e q, 0}$ and $W_{i n, n}=0$ for $n \neq 0$. We set $n=4$ in the structure Equation (112) in Appendix F (for $W_{e q}$ ). Then:

$$
\tilde{W}_{4}(s)=D_{Q, 4}[n=4 ; s] \sum_{n^{\prime}=1}^{3} B_{4, n^{\prime}}(s) \tilde{W}_{n^{\prime}}(s)
$$


The operators, $B_{4, n^{\prime}}$, follow by comparison with Equation (112). The $\hbar$-dependent quantum corrections in Equation (41) are responsible for the fact that $B_{4,2}(s)$ and $B_{4,1}(s)$ are non-vanishing, in principle. The long-time approximation in Section 2.2 for generic $W_{c, s t}=W_{c . e q}$ can be generalized formally to the present quantum case as follows. By arguing that the $t$-dependence of $W_{4}$ is slaved approximately by those of $W_{n}$, for $n=0,1,2,3$, one replaces $D_{Q, 4}[n=4 ; s]$, all $D_{Q, 4}\left[n^{\prime \prime} ; s\right]\left(n^{\prime \prime}>4\right)$ and $B_{4, n^{\prime}}(s)$ with $n^{\prime}=1,2,3$, by $s$-independent operators, $D_{Q, 4}[4], D_{Q, 4}\left[n^{\prime \prime}\right]$ and $B_{4, n^{\prime}}$, respectively (say, by fixing $s=\epsilon>0$ ). Then, Equation (67) could be approximated as: $\tilde{W}_{4}(s) \simeq D_{Q, 4}[4] \sum_{n^{\prime}=1}^{3} B_{4, n^{\prime}} \tilde{W}_{n^{\prime}}(s)$. The latter, together with Equation (65), as they stand for $n=0,1,2,3$ (without setting $s=\epsilon$ ), would constitute a finite closed system for $\tilde{W}_{n}(s), n=0,1,2,3,4$, and would complete the approximation scheme. However, notice that neither Hermiticity nor non-negativity properties have been discussed nor invoked for the generalized operator continued fractions.

Next, we turn to the three-term quasiclassical hierarchy for $W_{e q}$ with Equation (66), considered in the preceding subsection. A long-time approximation can be formulated formally, like in Section 2.2. Due to the quantum correction at order $\hbar^{2}$, that hierarchy does not fulfill the requirement that $M_{n+1, n}$ be the adjoint of $-M_{n, n+1}$. We have been unable to transform such a hierarchy into another three-term one satisfying the adjointness requirement. Due to the latter difficulty, the actual counterpart of the continued fraction operator, $D[n ; s]$ (Sections 2.2 and 2.4), cannot be warranted to fulfill Hermiticity and non-negativity. Then, the argument employed in the purely classical case for $W_{c, e q}$ (Section 2.2) cannot be invoked to justify the approach to thermalization in the quasiclassical regime.

For $V$ 's in the first class and $W_{e q}$, similar formal constructions could be generalized for Equations (63) and (64) to all orders in $\hbar$ and finite $T$ ). Criticisms for them would also apply, similar to the above ones for $V$ 's in the second class and for $W_{e q}$, and contrary to the classical case.

On physical grounds, and in spite of our limitations to provide justifications, we expect that, just for $W_{e q}$ and $V$ 's in both classes, the long-time approximations outlined above for the moment approach give an approach to thermalization in the quantum regime for all hierarchies discussed in this subsection. The justification lies outside our scope here.

We shall discuss the inclusion of non-negligible dissipation due to the external $h b$ in the quantum case. Two non-equilibrium dissipative quantum master equations for $V$ 's in the second class (quartic potentials) have been dealt with in [20]. In the first one, $W_{e q}$ turned out to depend on dissipation, while the second was a specific, but quite interesting, subclass of Lindblad's theory [47], in which $W_{e q}$ equals the equilibrium Wigner function for the associated dissipationless Wigner equation with the same $V$. In both cases, we have studied the (more difficult) construction of the $H_{Q, n}$ 's and the non-equilibrium moments, $W_{n}$ 's, and hierarchies. The analysis in Section 2.2 (classical case) did justify that, at least, a very small (and, eventually, negligible) dissipation due to the $h b$ was consistent with Hermiticity and non-negativity and with the long-time approximation. In the actual quantum case, the above difficulties related to Hermiticity and non-negativity will also prevent the justification of the corresponding consistency. Nevertheless, it is physically natural to expect that in the actual open quantum system at finite $T$, the analysis with negligible friction will not lead to inconsistencies, and that is our standpoint in Sections 4-7.

While such consistencies refer to oversimplified open one-particle systems, long-time approximate evolutions for non-equilibrium closed large systems (without external $h b$ 's) are our main goal. The 
analysis in $[15,16,20]$ and Section 3 in the present work confirmed, a posteriori, the reliability of letting dissipation vanish and justified the long-time approximate evolution towards thermalization for closed classical large systems, precisely through an extension of the techniques employed for open classical one-particle systems with negligible friction. The pending issues are closed large quantum systems, which are far more difficult. Then, the extraction and awareness of the consequences from the neglecting of dissipation in an open quantum one-particle system could be, at least, potentially interesting.

\section{7. $V$ 's in the First Class: Large Thermal and de Broglie Wavelengths}

We shall suppose that $V \geq 0, V \rightarrow 0$ as $|x| \rightarrow+\infty$ and that it is finite and continuous, except for, at worst, a finite number of finite discontinuities. Let $T$ be suitably small, so that: (a) $k_{B} T \ll \operatorname{Max}(V)$ (the absolute maximum of $V(x)$, as $x$ varies); and (b) the thermal wavelength $\lambda_{t h}=\hbar[\beta / 2 m]^{1 / 2}$ is $\gg$ the size, $a$, of the domain where $V$ is appreciably different from zero. Let $q_{l} \equiv \hbar / \lambda_{t h}$. In what follows, we shall restrict to $W(x, q ; t) \simeq W_{l}(x, q ; t)=W_{l}$ for $|x| / a \gg 1$ and $|q|<q_{l}$, which implies $\hbar /|q|$ adequately larger than $a$ (large de Broglie wavelength). For $|q|<q_{l}$, the detailed structure of $V$ should not be relevant, as a leading approximation, for $W_{l}$. Then, the expansion of $M_{Q}$ in Equation (41) into successive derivatives of $V$, even if correct, does not seem adequate; one should take into account the integral form in Equation (41).

We shall be interested in the equilibrium Wigner function $W_{e q}(x, q) \simeq W_{l, e q}(x, q)$, given by Equations (39) and (47) and describing thermal equilibrium with the $h b$, in the actual low- $T$ regime characterized by the above Conditions (a) and (b). This is exemplified for the case of a finite repulsive square well in Appendix D. See Appendix G for the derivation of an approximate representation for $W_{e q}(x, q)$ for the above general $V$. To leading order (orders $\left(a / \lambda_{t h}\right)^{0}$ and $\left.\left((\beta M a x(V))^{-1 / 2}\right)^{0}\right)$, the underlying physics appears to correspond to total reflection and zero transmission of the quantum particle by the potential $V(x)$ concentrated in a region of very small size, $a$. This picture is qualitatively consistent with the general features of quantum bosonic many-particle systems at very low $T$ [48]. ¿From Appendices $\mathrm{G}$ and D, one gets:

$$
\begin{gathered}
W_{e q}(x, q) \simeq W_{l, e q}(x, q) \simeq \int_{0}^{\lambda_{t h}^{-1}} \frac{d k_{1}}{\pi \hbar} \exp \left[-\beta\left(\hbar k_{1}\right)^{2} / 2 m\right]\left[-2 \cos 2 k_{1} x \frac{\sin 2(q / \hbar) x}{q / \hbar}+\right. \\
\left.\frac{\sin 2\left(k_{1}+q / \hbar\right) x}{k_{1}+q / \hbar}+\frac{\sin 2\left(-k_{1}+q / \hbar\right) x}{-k_{1}+q / \hbar}\right]
\end{gathered}
$$

This $W_{l, e q}(x, q)$ can be $<0$ in some region, while, on the other hand, $\int_{|q|<q_{l}} d q W_{l, e q}(x, q)>0$. One has:

$$
a^{2} q \frac{\partial W_{l, e q}(x, q)}{\partial x} \simeq \int_{0}^{\lambda_{t h}^{-1}} \frac{d k_{1}}{\pi \hbar} \exp \left[-\beta\left(\hbar k_{1}\right)^{2} / 2 m\right]\left[4\left(k_{1} a\right)(q a) \sin 2 k_{1} x \frac{\sin 2(q / \hbar) x}{q / \hbar}\right]
$$

which is negligible to leading order. Then, under the above Conditions (a) and (b) and the approximations yielding Equation (68), it would appear that, to leading order, $M_{Q}$ in Equation (43) could be neglected. Moreover, $M_{Q}$ can be also neglected to leading order in the non-equilibrium Equation (41). In fact, in the integral form of $M_{Q} W$, we set $\exp \left[i 2\left(q-q^{\prime}\right) y / \hbar\right] \simeq 1$, under the above approximations, and notice that $\int_{-\infty}^{+\infty} d y[V(x+y)-V(x-y)]=0$. Then, one gets the quantum non-equilibrium equation:

$$
\frac{\partial W_{l}(x, q ; t)}{\partial t} \simeq-\frac{q}{m} \frac{\partial W_{l}(x, q ; t)}{\partial x}
$$


which is formally similar to the one encountered in the classical case, for $V=0$. We shall introduce the polynomials $H_{Q, l, n}=H_{Q, l, n}(y)(n=0,1,2,3, \ldots)$, orthogonalized in $y=q / q_{l}$ with weight function $W_{l, e q}(x, q)$ using Equation (49), by integrating in $|y| \leq+1$. New non-equilibrium moments, $W_{l, n}$ ( $n=0,1,2, \ldots)$, are introduced through the analogue of Equation (56), also by integrating in $|y| \leq+1$. With the corresponding replacements $\left(\epsilon_{Q, n, n-j} \rightarrow \epsilon_{Q, l, n, n-j}\right.$ and $\left.W_{n} \rightarrow W_{l, n}\right)$, Equations (58)-(62) now become a linear hierarchy for the new moments, $W_{l, n}$, in which all successive derivatives of $V$ are neglected to leading order. Moreover, further computation, also with $M_{Q}=0$, gives another three-term equation:

$$
\frac{\partial W_{l, 5}}{\partial t}=-\frac{q_{0}}{m} \frac{\partial W_{l, 6}}{\partial x}+\frac{q_{0}}{m} \frac{\partial}{\partial x}\left[\left(\epsilon_{Q, l, 6,4}-\epsilon_{Q, l, 5,3}\right) W_{l, 4}\right]+\frac{q_{0}}{m} \frac{\partial \epsilon_{Q, l, 5,3}}{\partial x} W_{l, 4}
$$

See Appendix G. It is plausible that the equations for all $\partial W_{l, n} / \partial t$ could constitute an infinite three-term hierarchy for any $n$ and $V=0$, namely:

$$
\frac{\partial W_{l, n}}{\partial t}=-\frac{q_{0}}{m} \frac{\partial W_{l, n+1}}{\partial x}+\frac{q_{0}}{m} \frac{\partial}{\partial x}\left[\left(\epsilon_{Q, l, n+1, n-1}-\epsilon_{Q, l, n, n-2}\right) W_{l, n-1}\right]+\frac{q_{0}}{m}\left[\frac{\partial \epsilon_{Q, l, n, n-2}}{\partial x}\right] W_{l, n-1}
$$

for a generic $W_{s t}$, but a proof lies outside our scope here.

For the approximate hierarchy Equation (72) in the actual quantum system, under the above Conditions (a) and (b) and approximations, a long-time approximation can also be formulated formally, by following the procedure in Section 2.4. Approximate estimates in Appendix G suggest that those $H_{Q, l, n}$ are not radically different (or differ little, eventually) from those determined by the Gaussian $\exp \left[-\frac{\beta q^{2}}{2 m}\right]$, for very large $\beta$ and $|q|<q_{l}$. The Gaussian $\exp \left[-\frac{\beta q^{2}}{2 m}\right]$ (for any $|q|<+\infty$ ) determines a three-term non-equilibrium hierarchy similar to the one for $g_{n}$ 's in Equation (6) with $V=0$, which displays $D$ 's with Hermiticity and non-negativity and, so, thermalization under the long-time approximation. The latter $D$ 's could possibly not differ much from those for Equation (72). Then, for the approximate hierarchy Equation (72), an approximate long-time thermalization with the $h b$ could possibly be justified. Detailed computations lie outside our scope.

\section{Conclusions and Discussion}

The existence of approximate long-time thermalization or the absence thereof for open one-dimensional systems subject to a potential $V$ and to a $h b$ (with negligible external dissipation) has been studied, in the framework of hierarchies for non-equilibrium moments $\left(W_{c, n}\right.$ and $\left.W_{n}\right)$. The conceptual issues involved in treating an open system subject to a $h b$ with negligible dissipation have been discussed (Sections 2.3 and 6.4). The universal dependences of the non-equilibrium Liouville and Wigner equations on momenta imply that the equations for $W_{c, n}$ and $W_{n}$ have universal dependences on one unique moment of higher order ( $W_{c, n+1}$ and $W_{n+1}$, respectively), while in different cases, there are various dependences on moments of lower orders.

In the classical case, the non-equilibrium moments, $W_{c, n}$, for the canonical distribution, $W_{c, e q}$, are obtained in terms of operator continued fractions, D's, which are Hermitian and have non-negative eigenvalues (non-negativity): these properties, in a suitable long-time approximation, justify thermalization. In this work, we deal with a general stationary $W_{c, s t}\left(\neq W_{c, e q}\right)$, which is not Gaussian in momenta and fails, on physical grounds, to lead to approximate long-time thermalization, and the 
moment method displays this failure. In fact, hierarchies for the non-equilibrium moments, $W_{c, n}$ 's, determined by $W_{c, s t}$ are obtained for $n \leq 4$, but they lead to several unsolved difficulties (Section 2.4). Then, the formal construction through non-equilibrium moments provides one additional partial support to the selection of the Boltzmann equilibrium distribution, $W_{c, e q}$, out of the set of all $W_{c, s t}$, on a dynamical basis (although not a proof of it). From the outset, $W_{c, e q}$ and $W_{c, s t}$ correspond to different situations, and the moment method emphasizes, a posteriori, the differences. The microcanonical $W_{c, s t}$ and its special features are discussed briefly. The above analysis is one novelty of the present paper. The formal structure of the non-equilibrium hierarchies for $n \leq 4$ so obtained is very useful for the quantum case.

For closed classical interacting non-relativistic many-particle systems, without external $h b$ 's and for the canonical equilibrium distribution, $W_{c, e q}$, non-equilibrium three-term hierarchies for moments with Hermiticity have enabled us to justify approximate long-time thermalization $[15,16,20]$. Here, we have pointed out an interpretation in terms of interacting random walkers and an approximate Lyapunov function yielding an arrow of time, as novelties. Our proposal, based directly on moment approaches to classical non-equilibrium statistical mechanics and selecting approximately a time direction in the evolution, fails to give a non-equilibrium entropy. It could possibly be regarded as partially complementary to the proposals by other authors. The latter do not appear to be based directly on moment methods in classical non-equilibrium statistical mechanics, but, on the other hand, they do provide non-equilibrium entropies and other ways to select a time arrow, in different settings.

We have concentrated on a non-equilibrium open quantum one-dimensional particle, in the presence of a $h b$ at thermal equilibrium at $T$ with negligible external dissipation and subject to suitable repulsive $V(x)$ 's (characterized in Section 4.1). We have used non-equilibrium Wigner functions $W(x, q ; t)$, to all orders in Planck's constant.

The following developments and results are reported in this work (and are new, to the best of the author's knowledge).

The exact equilibrium canonical Wigner function, $W_{e q}(x, q)$, for a repulsive finite square potential is presented.

We have shown that $W_{e q}(x, q)>0$ for small $T, x$ and $q$ and have obtained two exact relationships for any non-equilibrium, $W$.

We have assumed that, for rather general repulsive $V$ 's, any $W$ and any stationary Wigner functions, $W_{s t}$ (even if $<0$ in some region), determine quasi-definite functionals [26] regarding their dependences on the momentum, $q$. The assumption is confirmed for the $W_{s t}(<0$ in some domain) corresponding to the first excited state of the quantum harmonic oscillator. That general assumption implies the existence of orthogonal polynomials, $H_{Q, n}$, in $q$ 's, having $W_{s t}$ 's as weight functions, and leads to non-equilibrium moments, $W_{n}$, of $W$.

Non-equilibrium quantum hierarchies for the $W_{n}$ 's have been obtained. As an illustration, we treat the $H_{Q, n}$ 's and the hierarchy for the first excited state of the harmonic oscillator. In general, with $V \neq 0$, the hierarchies for each $W_{n}$ are not three-term ones, due to moments $W_{n^{\prime}}$ with $n^{\prime}<n$, neither for a generic $W_{s t}$ nor for $W_{e q}$, due to the quantum corrections. As another illustration, we have studied a non-equilibrium four-term hierarchy and have outlined its solution in terms of generalized operator continued fractions. 
As an interesting new check for $W_{s t}=W_{e q}$, we have found consistency between the present non-equilibrium quantum hierarchies and the three-term ones derived previously [21] in the quasiclassical regime at high temperature (up to and including order $\hbar^{2}$ ).

In the non-equilibrium hierarchies for the corresponding $W_{e q}$ 's (both in the full quantum case and in the quasiclassical regime, up to and including order $\hbar^{2}$ ), we have formulated a long-time approximation. One expects physically that such an approximation should yield thermalization with the $h b$. However, we have been unable to display properties like Hermiticity and non-negativity and, hence, to justify thermalization with the $h b$, so far.

In the regime of large thermal and de Broglie wavelengths and to leading order, we have obtained an approximate $W_{e q}$ and an approximate non-equilibrium Wigner equation (Equations (68) and (70)) with $V \simeq 0$. To leading order, the underlying physics corresponds to total reflection and zero transmission of the quantum particle by the potential $V\left(x^{\prime}\right)$ concentrated in a region of very small size. Further computations with $V \simeq 0$ show that the equation for the non-equilibrium moment $W_{5}$ contains a three-term structure. That and the hierarchy for $n \leq 4$ obtained in Section 6.1 suggest that, for $V \simeq 0$, the non-equilibrium quantum hierarchy could plausibly be a three-term one for any $n$. An approximate long-time evolution towards thermalization through moment methods can also be formulated. Based upon that, we have argued that, in such a regime, an approximate long-time thermalization could possibly hold.

Among other pending problems, we quote the following ones. In the classical case: (i) mathematical studies of the operator continued fractions, $D$ 's, in the three-term hierarchies for $W_{c, e q}$, for one and $N$ particles; (ii) connections of the non-equilibrium hierarchy for $N$ particles, in the long-time approximation, with non-equilibrium thermodynamics and fluid dynamics. There is no attempt here to replace the latter by moment methods: rather, non-equilibrium moments emphasize Brownian motion features and approximate long-time thermalization, and it could be interesting to relate those (eventually, complementary) standpoints. In the quantum case: (iii) for one particle, studies of the non-equilibrium hierarchies, which are not three-term ones, and of the generalized operator continued fractions; (iv) generalizations to closed quantum (at least, bosonic) many-particle systems, without external $h b$ 's, which are far more difficult and, hopefully, could receive some qualitative partial hints from the analysis in the present paper.

\section{Acknowledgments}

The author is grateful to the Editorial Office of Entropy and to the Guest Editor, Gian Paolo Beretta, for inviting him to contribute to the Special Issue Advances in Methods and Foundations on Non-Equilibrium Thermodynamics. The author acknowledges the financial support of Project FIS2012-35719-C02-01, Ministerio de Economia y Competitividad, Spain. He is an associate member of BIFI (Instituto de Biocomputacion y Fisica de los Sistemas Complejos), Universidad de Zaragoza, Zaragoza, Spain. Several discussions with Gabriel F. Calvo on the dynamics based either upon random walkers or on average densities have been quite useful. 


\section{Conflicts of Interest}

The authors declare no conflicts of interest.

\section{Appendix}

A. $\epsilon_{c, n, n-j}$ 's for any $\boldsymbol{W}_{c, s t}$ and for $\boldsymbol{W}_{c, s t}=\boldsymbol{W}_{c, m c}$

We shall give the nonvanishing $\epsilon_{c, n, n-j}$ for low order $n=2,3,4,5$ ( $j$ even), by using Equations (2) and (20). After some algebra, one finds:

$$
\begin{aligned}
\epsilon_{c, 2,0}= & -<y^{2}>, \epsilon_{c, 3,1}=-\frac{<y^{4}>}{<y^{2}>} \\
\epsilon_{c, 4,2}= & \frac{<y^{2}><y^{4}>-<y^{6}>}{<y^{4}>-<y^{2}>^{2}}, \epsilon_{c, 4,0}=\frac{<y^{2}><y^{6}>-<y^{4}>^{2}}{<y^{4}>-<y^{2}>^{2}} \\
\epsilon_{c, 5,3}= & \frac{<y^{4}><y^{6}>-<y^{2}><y^{8}>}{<y^{2}><y^{6}>-<y^{4}>^{2}}, \epsilon_{c, 5,1}=\frac{<y^{4}><y^{8}>-<y^{6}>^{2}}{\left.<y^{6}><y^{2}>-<y^{4}\right\rangle^{2}} \\
<y^{n}>= & \frac{\int_{-\infty}^{+\infty} d y W_{c, s t}(x, q) y^{n}}{\int_{-\infty}^{+\infty} d y W_{c, s t}(x, q)}
\end{aligned}
$$

Equations (73)-(76) imply the following identities:

$$
\begin{aligned}
& \epsilon_{c, 4,0}+\epsilon_{c, 2,0}\left(-\epsilon_{c, 4,2}+\epsilon_{c, 3.1}\right)=0 \\
& \epsilon_{c, 5,1}-\epsilon_{c, 4,0}+\epsilon_{c, 3,1}\left(-\epsilon_{c, 5,3}+\epsilon_{c, 4,2}\right)=0 \\
& -\epsilon_{c, 2,0} \frac{\partial \epsilon_{c, 3,1}}{\partial x}+\left(3 \epsilon_{c, 2,0}-\epsilon_{c, 3,1}\right) \frac{m}{q_{0}^{2}} \frac{\partial V}{\partial x}=0 \\
& \frac{\partial \epsilon_{c, 4,0}}{\partial x}-\epsilon_{c, 3,1} \frac{\partial \epsilon_{c, 4,2}}{\partial x}+\left(4 \epsilon_{c, 3,1}-2 \epsilon_{c, 4,2}\right) \frac{m}{q_{0}^{2}} \frac{\partial V}{\partial x}=0
\end{aligned}
$$

\section{B. Classical Microcanonical Distribution $\boldsymbol{W}_{c, m c}$}

We consider two possible values for the total energy: $E(>0)$ and $E+\Delta(>0)$, with $E \gg \Delta>0$. First, we shall treat an open classical one particle system. The stationary microcanonical distribution describing equal probability for the particle, provided that $E \leq H_{c} \leq E+\Delta$, is:

$$
W_{c, m c}=\int_{E}^{E+\Delta} d E^{\prime} \delta\left(H_{c}-E^{\prime}\right)=\theta\left(H_{c}-E\right) \theta\left(E+\Delta-H_{c}\right)
$$

$\delta$ and $\theta$ denote, respectively, the Dirac delta and the step functions. We introduce some fixed ( $x$-independent) momentum, $q_{0}$. In order to have a finite weight function, $W_{c, m c}$, and a well-defined family of orthogonal polynomials $H_{c, n}(y)=H_{c, m c}(y)$ (in $y=q / q_{0}$ ), it is necessary that $\Delta \neq 0$. The coefficients, $\epsilon_{c, m c, n, n-j}$, in Equation (20) for $H_{c, m c}(y)$ are given by the above formulas in Appendix A, in terms of the corresponding $\left.<y^{n}\right\rangle=<y^{n}>_{m c}$ evaluated, in turn, with $W_{c}=W_{c, m c}$. One gets:

$$
\begin{aligned}
& \int_{-\infty}^{+\infty} d y W_{c, m c}(x, q)=\frac{4(2 m)^{1 / 2}(E-V(x))^{1 / 2}\left[\left(1+\Delta_{m c}\right)^{1 / 2}-1\right]}{q_{0}} \\
& \int_{-\infty}^{+\infty} d y W_{c, m c}(x, q) y^{n}=\frac{4(2 m)^{(n+1) / 2}(E-V(x))^{(n+1) / 2}\left[\left(1+\Delta_{m c}\right)^{(n+1) / 2}-1\right]}{(n+1) q_{0}^{n+1}}
\end{aligned}
$$


$n=2,4,6, \ldots$, with $\Delta_{m c}=\Delta /(E-V(x))$. The lowest nontrivial coefficients $C_{c, n}=C_{c, m c, n}$ in the counterpart of the fundamental recurrence relation Equation (21) for $H_{c, m c, n}(y)$ are:

$$
C_{c, m c, 2}=\frac{\epsilon_{c, m c, 2,0}-\epsilon_{c, m c, 3,1}}{q_{0}^{2}}, C_{c, m c, 3}=\frac{\epsilon_{c, m c, 3,1}-\epsilon_{c, m c, 4,2}}{q_{0}^{2}}, C_{c, m c, 4}=\frac{\epsilon_{c, m c, 4,2}-\epsilon_{c, m c, 5,3}}{q_{0}^{2}}
$$

The general theorem [26] requires that $C_{c, m c, n}>0$. This general requirement is warranted, provided that $\Delta>0$. Since $\Delta$ is small, by expanding into powers of $\Delta_{m c}$, one finds, for instance:

$$
\epsilon_{c, m c, 2,0}-\epsilon_{c, m c, 3,1}=2 m(E-V) \frac{37 \Delta_{m c}^{2}}{48}, \epsilon_{c, m c, 3,1}-\epsilon_{c, m c, 4,2}=2 m(E-V)\left(1+\frac{\Delta_{m c}}{2}\right)
$$

The first Equation (85) clearly shows that the fundamental recurrence relation and, hence, the very family of $H_{c, m c, n}(y)$ 's become ill-defined if $\Delta=0$. The non-equilibrium hierarchy for the moments determined by the family, $H_{c, m c}(y)$, could face the problems discussed in Section 2.4.

One now turns briefly to $N$ particles. The stationary classical microcanonical distribution and orthogonal polynomials can be obtained directly, by generalizing Equations (81)-(83), by using the Hamiltonian, $H_{c, N}$, in Section 3. The most interesting situation occurs when the number of degrees of freedom is very large (in the thermodynamical limit) and the classical microcanonical and canonical distributions yield the same equilibrium thermodynamics. The (rather delicate) analysis, based upon the equilibrium partition functions, requires that the system be divided into two subsystems, one being much smaller than the other (which behaves as a $h b$ ); see [48,49]. A study of the equilibrium distributions themselves (and their orthogonal polynomials) and of the off-equilibrium evolutions, in the transition from the microcanonical ensemble to the canonical one (under those thermodynamical limit conditions), lies outside our scope here. Since both ensembles give the same equilibrium thermodynamics, one pragmatic standpoint could be to concentrate primarily on the off-equilibrium evolution in the canonical ensemble; that is the point of view adopted here.

\section{Behavior of $\boldsymbol{W}_{\text {eq }}$ for Large $\boldsymbol{q}$}

Here, we shall check the behavior of $W_{e q}$ as $|q| \rightarrow$, assumed in Section 4.1. A quick decrease of $W_{e q}$ 's is necessary for constructing the orthogonal polynomials, $H_{Q, n}(y)$ 's. We shall always start from $\int_{-\infty}^{+\infty} d q q^{n} W_{e q}(x, q)$ for $n=0,1,2,3, \ldots$, and use Equations (39) and (47). First, we shall treat the case of harmonic/anharmonic oscillators ( $V$ 's in second class), for which all $\varphi_{j}(y)$ 's and all $\left[d^{n} \varphi_{j}(y) / d y^{n}\right]$ are continuous and finite. By exchanging $\int_{-\infty}^{+\infty} d q$ and $\int_{-\infty}^{+\infty} d y$ and by performing first $\int_{-\infty}^{+\infty} d q$, we get:

$$
\int_{-\infty}^{+\infty} d q q^{n} W(x, q)=\left[\frac{-i \hbar}{2}\right]^{n} \sum_{j} \exp \left[-\beta E_{j}\right] \int_{-\infty}^{+\infty} d y\left[\varphi_{j}(x-y) \varphi_{j}(x+y)^{*}\right]\left[\frac{\partial}{\partial y}\right]^{n} \delta(y)
$$

$\delta(y)$ being the Dirac delta function. One can integrate directly by using $\delta(y)$ :

$$
\int_{-\infty}^{+\infty} d q q^{n} W(x, q)=\left[\frac{i \hbar}{2}\right]^{n} \sum_{j} \exp \left[-\beta E_{j}\right]\left[d^{n}\left(\varphi_{j}(x-y) \varphi_{j}(x+y)^{*}\right) / d y^{n}\right]_{y=0}
$$

$\sum_{j}$ denoting an infinite sum. $\exp \left[-\beta E_{j}\right]$ in Equation (87) decreases quickly with $j$. Then, the infinite series Equation (87) converges and $\int_{-\infty}^{+\infty} d q q^{n} W_{e q}(x, q)$ is finite for any $x$ and any $n=1,2,3 \ldots$ Next, we 
consider $V$ 's in the first class and study two possibilities: (a) Let $V(x)$ and all $d^{n} V(x) / d x^{n}, n=1,2,3 \ldots$, be continuous for any $x$, so that all $\varphi_{j}(y)$ 's and all $\left[d^{n} \varphi_{j}(y) / d y^{n}\right]$ are continuous. Then, by operating like for $V$ 's in the second class above, we get:

$$
\int_{-\infty}^{+\infty} d q q^{n} W(x, q)=\left[\frac{i \hbar}{2}\right]^{n} \sum_{j} \exp \left[-\beta E_{j}\right]\left[d^{n}\left(\varphi_{j}(x-y) \varphi_{j}(x+y)^{*}\right) / d y^{n}\right]_{y=0}
$$

However, now, $\sum_{j} \equiv \int_{-\infty}^{+\infty} d j$, and $E_{j}$ sweeps the continuous positive real half-line: $0 \leq E_{j}<+\infty$, with a double degeneracy, associated with two different asymptotic conditions (incoming plane waves) at $x \rightarrow$ $\pm \infty$, with the same energy, $E_{j}$. Then, Equation (87) also converges and renders $\int_{-\infty}^{+\infty} d q q^{n} W_{e q}(x, q)$ finite for any $x$ and any $n=1,2,3 \ldots$ (b) Let $V$ and all $d^{n} V(x) / d x^{n}, n=1,2,3 \ldots$, be continuous for almost any $x$, except for a finite number of points $x=a_{l}, l=1, \ldots, l_{\max }<\infty$, at which $V$ has finite discontinuities. Then, one also arrives to the same convergent result in Equation (88) for any $n=1,2,3 \ldots$, provided that $x \neq a_{l}$. The finiteness of $\int_{-\infty}^{+\infty} d q q^{n} W_{e q}(x, q)$ for any $n=1,2,3 \ldots$ is not warranted at $x=a_{l}$, due to the discontinuities of $V$ and, hence, of derivatives of $\varphi_{j}$ 's (of orders $\geq 2$ ) at the latter points.

\section{D. $W_{\text {eq }}$ for a Finite Repulsive Square Well}

We shall consider the finite repulsive square well: $V(x)=+V_{0}>0$, for $|x|<a / 2$ and $V(x)=0$, for $|x|>a / 2$, with $V_{0}<+\infty$ and $0<a<+\infty$. We shall give the eigenfunctions of $H$, Equation (37). The first set of eigenfunctions is $\left(j=k_{1} \geq 0, E_{j}=\left(\hbar k_{1}\right)^{2} / 2 m, A=(2 \pi)^{-1 / 2}\right)$ :

$$
\begin{aligned}
& \varphi_{+, k_{1}}(x)=A\left[\exp i k_{1} x+A_{1}\left(k_{1}\right) \exp \left(-i k_{1} x\right)\right], x<-a / 2 \\
& \varphi_{+, k_{1}}(x)=A\left[A_{2}\left(k_{1}\right) \exp i k_{2} x+A_{3}\left(k_{1}\right) \exp \left(-i k_{2} x\right)\right],-a / 2<x<+a / 2 \\
& \varphi_{+, k_{1}}(x)=A A_{4}\left(k_{1}\right) \exp i k_{1} x, x>a / 2
\end{aligned}
$$

with $k_{2}=k_{2}(E)=\left[2 m\left(E-V_{0}\right)\right]^{1 / 2} / \hbar$. If $E-V_{0}>0$, then $k_{2}>0$, while, if $E-V_{0}<0$, then $k_{2}=+i \kappa_{2}$, with $\kappa_{2}>0$. The amplitudes $A_{l}=A_{l}\left(k_{1}\right), l=1, \ldots, 4$, are:

$$
\begin{aligned}
& A_{1}=\frac{\left(k_{2}^{2}-k_{1}^{2}\right) \exp \left(-i k_{1} a\right)\left(\exp \left(i k_{2} a\right)-\exp \left(-i k_{2} a\right)\right)}{D} \\
& A_{2}=\frac{2 k_{1}\left(k_{1}+k_{2}\right) \exp \left[-i\left(k_{1}+k_{2}\right)(a / 2)\right]}{D}, A_{3}=\frac{2 k_{1}\left(-k_{1}+k_{2}\right) \exp \left[i\left(-k_{1}+k_{2}\right)(a / 2)\right]}{D} \\
& A_{4}=\frac{4 k_{1} k_{2} \exp \left(-i k_{1} a\right)}{D}, D=\left(k_{1}+k_{2}\right)^{2} \exp \left(-i k_{2} a\right)-\left(-k_{1}+k_{2}\right)^{2} \exp i k_{2} a
\end{aligned}
$$

The second set of eigenfunctions is $\left(j=-k_{1} \leq 0, E_{j}=\left(\hbar k_{1}\right)^{2} / 2 m\right.$ :

$$
\begin{aligned}
& \varphi_{-, k_{1}}(x)=A\left[\exp \left(-i k_{1} x\right)+B_{1}\left(k_{1}\right) \exp i k_{1} x\right], x>a / 2 \\
& \varphi_{-, k_{1}}(x)=A\left[B_{2}\left(k_{1}\right) \exp \left(-i k_{2} x\right)+B_{3}\left(k_{1}\right) \exp i k_{2} x\right],-a / 2<x<+a / 2 \\
& \varphi_{-, k_{1}}(x)=A B_{4}\left(k_{1}\right) \exp \left(-i k_{1} x\right), x<-a / 2
\end{aligned}
$$

with the same $A$ and the same $k_{2}=k_{2}(E)$ as for the first set. One gets: $B_{l}\left(k_{1}\right)=A_{l}\left(k_{1}\right)$. The equilibrium Wigner function in Equations (39)-(47) becomes:

$$
\begin{aligned}
W_{e q}(x, q)= & \frac{1}{\pi \hbar} \int_{-\infty}^{+\infty} d y \exp \left[\frac{i 2 q y}{\hbar}\right] \int_{0}^{+\infty} d k_{1} \exp \left[-\beta\left(\hbar k_{1}\right)^{2} / 2 m\right]\left[\varphi_{+, k_{1}}(x-y) \varphi_{+, k_{1}}(x+y)^{*}+\right. \\
& \left.\varphi_{-, k_{1}}(x-y) \varphi_{-, k_{1}}(x+y)^{*}\right]
\end{aligned}
$$


By using Equations (92)-(94) and their counterparts for $B_{l}\left(k_{1}\right)$, one finds important exact cancellations. It will suffice to display the resulting exact $W_{e q}(x, q)$ for $x \geq 0$ :

$$
\begin{aligned}
& W_{e q}(x, q)=\frac{1}{\pi \hbar} \int_{0}^{+\infty} d k_{1} \exp \left[-\beta\left(\hbar k_{1}\right)^{2} / 2 m\right]\left[\int _ { - ( a / 2 ) - x } ^ { ( a / 2 ) + 2 x } d y \operatorname { e x p } [ \frac { i 2 q y } { \hbar } ] \left[\varphi_{+, k_{1}}(x-y) \varphi_{+, k_{1}}(x+y)^{*}+\right.\right. \\
& \left.\varphi_{-, k_{1}}(x-y) \varphi_{-, k_{1}}(x+y)^{*}\right]+\int_{-\infty}^{-(a / 2)-x} d y \exp \left[\frac{i 2 q y}{\hbar}\right]\left[B_{4}\left(k_{1}\right)^{*} \exp \left(2 i k_{1} y\right)+A_{4}\left(k_{1}\right) \exp \left(-2 i k_{1} y\right]+\right. \\
& \int_{a / 2)+2 x}^{+\infty} d y \exp \left[\frac{i 2 q y}{\hbar}\right]\left[B_{4}\left(k_{1}\right) \exp \left(2 i k_{1} y\right)+A_{4}\left(k_{1}\right)^{*} \exp \left(-2 i k_{1} y\right]\right]
\end{aligned}
$$

In agreement with Appendix $\mathrm{C}, \int_{-\infty}^{+\infty} d q q^{n} W_{e q}(x, q)$ is finite for any $n=1,2,3 \ldots$ and any $x \neq \pm a / 2$. Let us consider the regime of large thermal and de Broglie wavelengths: $\lambda_{t h}=\hbar(\beta / 2 m)^{1 / 2}, q_{l}=\hbar / \lambda_{t h},|q|$ $a \ll \hbar,|q|<q_{l}\left(\lambda_{t h} \gg a\right), V_{0} \gg \beta^{-1}$ and $|x| / a \gg 1$. Let $\kappa_{2,0}=\left[2 m V_{0}\right]^{1 / 2} / \hbar$. Then, the dominant contributions to $\int_{0}^{+\infty} d k_{1}$ in Equation (99) come from $k_{1} \lambda_{t h}<1$. We shall start by keeping terms only up to and including orders $a / \lambda_{t h}$ and $\left(\beta V_{0}\right)^{-1 / 2}$ and indicate later at which stage they are dropped, so as to have a record of them and to handle, and at the end, manageable expressions at leading order. Then: $A_{1} \simeq-1+i\left[k_{1} a+2\left(k_{1} / \kappa_{2,0}\right) \operatorname{coth}\left(a \kappa_{2,0}\right)\right]$, while $A_{2} \simeq\left(k_{1} / \kappa_{2,0}\right)\left(\exp \left(a \kappa_{2,0} / 2\right) / \sinh \left(a \kappa_{2,0}\right)\right.$, $A_{3} \simeq\left(k_{1} / \kappa_{2,0}\right)\left(\exp \left(-a \kappa_{2,0} / 2\right) / \sinh \left(a \kappa_{2,0}\right)\right.$ and $A_{4} \simeq\left(k_{1} / \kappa_{2,0}\right)\left(2 / \sinh \left(a \kappa_{2,0}\right)\right.$. By starting from Equation (99): (i) the contributions from $A_{4}, A_{4}^{*}, B_{4}$ and $B_{4}^{*}$ (second and third integrals over $y$ ) are of order $k_{1} / \kappa_{2,0}$, as is that from $\int_{(a / 2)+x}^{(a / 2)+2 x} d y$; (ii) $\int_{-(a / 2)-x}^{(a / 2)+x} d y$ is dominated by $\int_{-x}^{+x} d y \varphi_{-, k_{1}} \varphi_{-, k_{1}}^{*}$ (dropping terms of order $\left(k_{1} / \kappa_{2,0}\right)^{2}$ and $\int_{-(a / 2)-x}^{-x} d y$ and $\int_{x}^{(a / 2)+x} d y$ ); (iii) in $\int_{-x}^{+x} d y \varphi_{-, k_{1}} \varphi_{-, k_{1}}^{*}$, we discard the two contributions of orders $k_{1} / \kappa_{2,0}$ and $k_{1} a$ and the one quadratic in them (all coming from $B_{1}+1$ and $\left.B_{1}^{*}+1\right)$. Then:

$$
\begin{aligned}
& W_{e q}(x, q) \simeq W_{l, e q}(x, q) \simeq \int_{0}^{\lambda_{t h}^{-1}} \frac{d k_{1}}{\pi \hbar} \\
& \times \exp \left[-\frac{\beta\left(\hbar k_{1}\right)^{2}}{2 m}\right]\left[-2 \cos 2 k_{1} x \frac{\sin 2(q / \hbar) x}{q / \hbar}+\frac{\sin 2\left(k_{1}+q / \hbar\right) x}{k_{1}+q / \hbar}+\frac{\sin 2\left(-k_{1}+q / \hbar\right) x}{-k_{1}+q / \hbar}\right]
\end{aligned}
$$

which corresponds to dominant reflection and small transmission of the quantum particle by the repulsive finite square well, at leading order (orders $\left(a / \lambda_{t h}\right)^{0}$ and $\left.\left(\left(\beta V_{0}\right)^{-1 / 2}\right)^{0}\right)$. One gets: $\int_{|q|<q_{l}} d q q^{2 n+1} W_{l, e q}(x, q)=0$, for $n=0,1,2,3, \ldots$ These results will be helpful in Section 7 and Appendix G.

\section{E. First Excited State of Harmonic Oscillator: Orthogonal Polynomials and Hierarchy}

First, we shall study whether $W_{s t}$, given in Equation (55), provides a quasi-definite functional regarding the $y$ dependence. We have computed the actual $\mu_{n}$ 's, the $(S+1) \times(S+1)$ matrices $M_{S}$ and their determinants: $\operatorname{Det}\left[M_{S}\right]$. One finds that $\operatorname{Det}\left[M_{S}\right]$ bears the structure $\exp \left[-(S+1)\left(2 x^{2}\right)\right] d_{S+1}$, where $d_{S+1}$ is a polynomial in $x^{2}$ of degree $S+1$, which can vanish only at a finite set of $x$ values. It follows from a theorem in [26] that $W_{s t}$ is a quasi-definite functional regarding the $y$ dependence for any $x$, except at a set of $x$-values having zero measure (formed by all zeroes of all $d_{S+1}$ 's). Consequently, 
except for the latter set, the orthogonal polynomials, $H_{Q, n}$, exist for the quasi-definite functional, $W_{s t}$. We shall give the lowest non-vanishing $\sigma_{n, n-j}$ ( $j$ being even) in those $H_{Q, n}$ 's (Equation (51)):

$$
\begin{aligned}
& \sigma_{2,0}=-\frac{1}{x^{2}}, \sigma_{3,1}=-\frac{3 !}{2 x^{2}+1} \\
& \sigma_{4,2}=-x^{2} \sigma_{4,0}, \sigma_{4,0}=-\frac{4 !}{4 x^{2}+4-\left(1 / 2 x^{2}\right)}
\end{aligned}
$$

Consistently with a general theorem in [26], the $H_{Q, n}$ 's fulfill the recurrence relation:

$$
2 y H_{Q, n}(y)=H_{Q, n+1}(y)+C_{n} H_{Q, n-1}(y)
$$

for $n=0,1,2, \ldots$, with $C_{0} \equiv 0 . C_{n}$ are $y$-independent (but $x$-dependent). The lowest $C_{n}$ 's are:

$$
\begin{aligned}
& C_{1}=2-\sigma_{2,0}, C_{2}=4+\sigma_{2,0}-\sigma_{3,1} \\
& C_{3}=6+\sigma_{3,1}-\sigma_{4,2}
\end{aligned}
$$

That general theorem in [26] does not warrant that all $C_{n}$ 's be positive; in fact, $C_{2}<0$ for small $x^{2}$.

We now consider the quantum Equation (53) for a non-equilibrium, $W$, and define its off-equilibrium moments, $W_{n}$, by using the actual counterpart of Equation (56) with the polynomials, $H_{Q, n}$, constructed in this Appendix. Through computations similar to those in Section 6.1, one gets the following offequilibrium three-term hierarchy for the $W_{n}$, for low $n$ 's:

$$
\begin{aligned}
& \frac{\partial W_{0}}{\partial t}=-\frac{1}{2^{3 / 2}} \frac{\partial W_{1}}{\partial x} \\
& \frac{\partial W_{1}}{\partial t}=-\frac{1}{2^{3 / 2}} \frac{\partial W_{2}}{\partial x}+\frac{1}{2^{3 / 2}} \frac{\partial}{\partial x}\left[\left(\sigma_{2,0}-2\right] W_{0}\right]-2^{1 / 2} 2 x W_{0} \\
& \left.\frac{\partial W_{2}}{\partial t}=-\frac{1}{2^{3 / 2}} \frac{\partial W_{3}}{\partial x}+\frac{1}{2^{3 / 2}} \frac{\partial}{\partial x}\left[\sigma_{3,1}-4\right) W_{1}\right]-\frac{\sigma_{2,0}}{2^{3 / 2}} \frac{\partial W_{1}}{\partial x}-2^{1 / 2} 4 x W_{1} \\
& \frac{\partial W_{3}}{\partial t}=-\frac{1}{2^{3 / 2}} \frac{\partial W_{4}}{\partial x}+\frac{1}{2^{3 / 2}} \frac{\partial}{\partial x}\left[\left(\sigma_{4,2}-6\right) W_{2}\right]-\frac{\sigma_{3,1}}{2^{3 / 2}} \frac{\partial W_{2}}{\partial x}-2^{1 / 2} 6 x W_{2}
\end{aligned}
$$

and so on for $W_{n}, n>3$, with suitable initial conditions $W_{i n, n}$. The computations for $n=3,4,5, \ldots$ become more difficult. The hierarchy in Equations (106)-(109) is satisfied by the $t$-independent moments determined by $W_{s t}$ in Equation (55). The actual hierarchy falls into the class given in Equation (27), for which $M_{s t, n, n+1}$ is not the adjoint of $-M_{s t, n+1, n}$. Then, the corresponding operator continued fractions (counterparts of Equation (28)) are not expected to fulfill Hermiticity and non-negativity properties; recall Section 2.4.

\section{F. Formal Solution of Equation (65) through Generalized Operator Continued Fractions}

The procedure for solving formally the four-term hierarchy Equation (65) (for any $n$ ) in terms of generalized operator continued fractions extends directly to the strategy employed for the three-term one, at the expense of working with more complicated expressions. For simplicity, we shall assume the initial condition $W_{i n, 0} \neq 0, W_{i n, 0} \neq W_{e q, 0}$ and $W_{i n, n}=0$ for $n \neq 0$. We perform the Laplace transform, which leads from Equation (65) to:

$$
s \tilde{W}_{n}(s)=W_{i n, n}-M_{n, n+1} \tilde{W}_{n+1}(s)-M_{n, n-1} \tilde{W}_{n-1}(s)-M_{n, n-3} \tilde{W}_{n-3}(s)_{n-3}
$$


The solution of Equation (110) is given in terms of products of the $s$-dependent generalized operator continued fractions, $D_{Q, 4}[n ; s]$. The latter are defined recurrently, for $n=1,2, \ldots$, through the following generalization of Equation (9):

$$
\begin{aligned}
D_{Q, 4}[n ; s]= & {\left[s I-M_{n, n+1} D_{Q, 4}[n+1 ; s]\left[M_{n+1, n}+M_{n+1, n+2} D_{Q, 4}[n+2 ; s] M_{n+2, n+3} \times\right.\right.} \\
& \left.\left.D_{Q, 4}[n+3 ; s] M_{n+3, n}\right]\right]^{-1}
\end{aligned}
$$

$I$ is the unit operator. By iteration of Equation (111), $D_{Q, 4}[n ; s]$ becomes a formal generalized infinite continued fraction of nonconmuting operators. Omitting calculational details, one gets for $n=1,2, \ldots$ :

$$
\begin{aligned}
\tilde{W}_{n}(s)= & D_{Q, 4}[n ; s]\left[\left[-M_{n, n+1} D_{Q, 4}[n+1 ; s] M_{n+1, n+2} D_{Q, 4}[n+2 ; s] M_{n+2, n-1}-M_{n, n-1}\right] \tilde{W}_{n-1}(s)+\right. \\
& \left.M_{n, n+1} D_{Q, 4}[n+1 ; s] M_{n+1, n-2} \tilde{W}_{n-2}(s)-M_{n, n-3} \tilde{W}_{n-3}(s)\right]
\end{aligned}
$$

\section{G. Approximate $\boldsymbol{W}_{e q}(\boldsymbol{x}, \boldsymbol{q})$ for Large Thermal and de Broglie Wavelengths}

We shall get an approximate representation for $W_{l, e q}(x, q)$ with $V$ 's in the first class, to leading order in the low- $T$ regime characterized by the Conditions (a) and (b) in Section 7. The exact $W_{e q}(x, q)$ (Equations (39) and (47)) is given, alternatively, by Equation (98), in which $\varphi_{+, k_{1}}(x)$ is now the exact solution of the following inhomogeneous linear integral equation for one-dimensional scattering in $-\infty<x<+\infty$, with the incoming plane wave as $x \rightarrow-\infty$ :

$$
\varphi_{+, k_{1}}(x)=\exp i k_{1} x-\frac{m i}{\hbar^{2} k_{1}} \int_{-a / 2}^{+a / 2} d x^{\prime} \exp i k_{1}\left|x-x^{\prime}\right| V\left(x^{\prime}\right) \varphi_{+, k_{1}}\left(x^{\prime}\right)
$$

In Equation (113) for simplicity, we set firstly $V\left(x^{\prime}\right) \neq 0$ only in $-a / 2<x^{\prime}<+a / 2$ (finite range potential). We shall study the approximate solution of Equation (113) for $-a / 2<x<+a / 2$ and very small $k_{1}$. That approximate solution cannot come from Equation (113) as it stands, due to the explicit singularity, $k_{1}^{-1}$, in the integral. The problem will be solved by extending to scattering a technique used in [50] for bound states. For that purpose, we set exp $i k_{1}\left|x-x^{\prime}\right|=1+C\left(k_{1}\left|x-x^{\prime}\right|\right)$ (thereby defining $C\left(k_{1}\left|x-x^{\prime}\right|\right)$ ), multiply the resulting Equation (113) by $V(x)$, integrate in $-a / 2<x<+a / 2$ and solve for $\int_{-a / 2}^{+a / 2} d x V(x) \varphi_{+, k_{1}}(x)$. By reshuffling the latter into Equation (113), we get for $-a / 2<x<+a / 2$ :

$$
\begin{aligned}
& \varphi_{+, k_{1}}(x)= \exp i k_{1} x-\frac{1}{\left(\hbar^{2} k_{1} / i m\right)+\int_{-a / 2}^{+a / 2} d x^{\prime \prime} V\left(x^{\prime \prime}\right)}\left[\int_{-a / 2}^{+a / 2} d x^{\prime} \exp i k_{1} x^{\prime} V\left(x^{\prime}\right)\right. \\
&\left.-\int_{-a / 2}^{+a / 2} d x^{\prime} \frac{m i V\left(x^{\prime}\right)}{\hbar^{2} k_{1}} \times \int_{-a / 2}^{+a / 2} d x^{\prime \prime} C\left(k_{1}\left|x^{\prime}-x^{\prime \prime}\right|\right) V\left(x^{\prime \prime}\right) \varphi_{+, k_{1}}\left(x^{\prime \prime}\right)\right] \\
&-\int_{-a / 2}^{+a / 2} d x^{\prime} \frac{m i C\left(k_{1}\left|x-x^{\prime}\right|\right)}{\hbar^{2} k_{1}} V\left(x^{\prime}\right) \varphi_{+, k_{1}}\left(x^{\prime}\right)
\end{aligned}
$$

Equation (115) has no singularity at $k_{1}=0$ (since $C\left(k_{1}\left|x-x^{\prime}\right|\right) / k_{1}$ is finite as $k_{1} \rightarrow 0$ ). Even if the iterations of Equation (115) provide $\varphi_{+, k_{1}}(x)$, the full corrections of order $k_{1}$ require one to solve a (singularity-free) linear integral equation, so that we shall limit ourselves to leading order $\left(\left(k_{1}\right)^{0}\right)$, which is simpler. We come back to Equation (113) for $x<-a / 2$, set $\exp \left(-i k_{1} x^{\prime}\right) \simeq+1$ for $-a / 2<x^{\prime}<$ 
$+a / 2$, use the above $\int_{-a / 2}^{+a / 2} d x V(x) \varphi_{+, k_{1}}(x)$ and see that the singularity in $k_{1}^{-1}$ cancels out, consistently with the study yielding Equation (115). In the resulting expression, we neglect the terms in $V\left(x^{\prime}\right) V\left(x^{\prime \prime}\right)$. For small $k_{1}$, the leading contribution for $x<-a / 2$ is: $\varphi_{+, k_{1}}(x) \simeq \exp i k_{1} x-\exp \left(-i k_{1} x\right)$, to order $\left(k_{1}\right)^{0}$ (the total reflection of the quantum particle by $V\left(x^{\prime}\right)$ ). A similar analysis for $x>a / 2$ gives: $\varphi_{+, k_{1}}(x) \simeq 0$, to order $\left(k_{1}\right)^{0}$ (zero transmission). There is a similar analysis for $\varphi_{-, k_{1}}(x)$ with the incoming plane wave as $x \rightarrow+\infty$. The same results hold for $V$ 's in the first class without finite range.

The above leading order approximations (to order $\left(k_{1}\right)^{0}$ ) apply in the low- $T$ regime characterized by the Conditions (a) and (b) in Section 7. Then, through Equation (98), the exact $W_{e q}(x, q)$ can be approximated like in the derivation of Equation (100) in Appendix D. The resulting expression is given in Equation (68). The new coefficients, $\epsilon_{Q, l, n, n-j}$, in the polynomials, $H_{Q, l, n}$, in Equation (49) are given formally by the right-hand sides of Equations (73)-(76) for low $n$ and $j$, with the above $W_{l, e q}(x, q)$. Equation (71) employed the new identities $\epsilon_{Q, l, 6,2}-\epsilon_{Q, l, 5,1}+\epsilon_{Q, l, 4,2}\left(-\epsilon_{Q, l, 6,4}+\epsilon_{Q, l, 5.3}\right)=0, \partial \epsilon_{Q, l, 5.3} / \partial x=0$ and $\partial \epsilon_{Q, l, 5.1} / \partial x=0$, which extend Equations (77)-(80).

For very large $x$, one can approximate Equation (68) by:

$$
\begin{aligned}
& W_{l, e q}(x, q) \simeq \int_{0}^{\lambda_{t h}^{-1}} \frac{d k_{1}}{\hbar} \exp \left[-\beta\left(\hbar k_{1}\right)^{2} / 2 m\right]\left[-2 \cos 2 k_{1} x \cdot \hbar \delta(q)+\delta\left(k_{1}+q / \hbar\right)+\right. \\
& \left.\delta\left(-k_{1}+q / \hbar\right)\right]=-\delta(q) \int_{0}^{\lambda_{t h}^{-1}} d k_{1} \exp \left[-\frac{\beta\left(\hbar k_{1}\right)^{2}}{2 m}\right] \cos 2 k_{1} x+\frac{1}{\hbar} \exp \left[-\frac{\beta q^{2}}{2 m}\right]
\end{aligned}
$$

$\delta$ being the Dirac delta function. By using Equation (115):

$$
\int_{|q|<q l} d q W_{l, e q}(x, q)=-\int_{0}^{\lambda_{t h}^{-1}} d k_{1} \exp \left[-\frac{\beta\left(\hbar k_{1}\right)^{2}}{2 m}\right] \cos 2 k_{1} x+\int_{-\lambda_{t h}^{-1}}^{\lambda_{t h}^{-1}} \frac{d q}{\hbar} \exp \left[-\frac{\beta q^{2}}{2 m}\right]>0
$$

Then, for very large $x$ and $|q| \leq q_{l}$ (except at $q=0$ ), $W_{e q}(x, q)$ equals roughly a Gaussian minus the $\delta(q)$ contribution. Even if $W_{l, e q}(x, q)$ in Equation (68) can be $<0$ in some region, a glance at it (and at the approximation for it in Equation (115)) would suggest that, in some sense and for $|q| \leq q_{l}$ and adequately large $x, W_{l, e q}(x, q)$ could have properties not radically different from those a Gaussian has in $q$ (in the same range). We use Equation (115)) below. Certain $\epsilon_{Q, l, n, n-j}\left(\epsilon_{Q, l, 3,1}, \epsilon_{Q, l, 5,3}, \epsilon_{Q, l, 5,1}, \ldots.\right)$ are $x$-independent and rather similar to those for the Gaussian case (say, to the classical case): the main difference is that, in the actual quantum case, one has structures like $\int_{-\lambda_{t h}^{-1}}^{\lambda_{t h}^{-1}}(d q / \hbar) \exp \left[-\left(\beta q^{2} / 2 m\right)\right]$, instead of the classical $\int_{-\infty}^{+\infty}(d q / \hbar) \exp \left[-\left(\beta q^{2} / 2 m\right)\right]$. Other $\epsilon_{Q, l, n, n-j}\left(\epsilon_{Q, l, 2,0}, \epsilon_{Q, l, 4,2}, \epsilon_{Q, l, 4,0}, \ldots.\right)$ are $x$-dependent: then one finds structures like $\int_{0}^{\lambda_{\text {th }}^{-1}} d k_{1} \exp \left[-\left(\beta q^{2} / 2 m\right)\right]\left[-\cos 2 k_{1} x+2\right]>0$, in which the $x$-dependent contributions are dominated by constant Gaussian-like ones.

\section{References}

1. Wallace, D. Reading list for advanced philosophy of physics: The philosophy of statistical mechanics. Available online: http://www.osti.gov/eprints/topicpages/documents/record/366/ 4717199.html (accessed on 20 Febraury 2014).

2. Kreuzer, H. J. Nonequilibrium Thermodynamics and its Statistical Foundations; Clarendon Press: Oxford, UK, 1981. 
3. Balescu, R. Equilibrium and Nonequilibrium Statistical Mechanics; John Wiley and Sons: New York, NY, USA, 1975.

4. Liboff, R.L. Kinetic Theory, 2nd ed.; John Wiley (Interscience): New York, NY, USA, 1998.

5. Zubarev, D.; Morozov V.G.; Röpke, G. Statistical Mechanics of Nonequilibrium Processes; Akademie Verlag: Berlin, Germany, 1996; Volume I.

6. Wigner, E.P. On the quantum correction for thermodynamic equilibrium. Phys. Rev. 1932, 40, 749-759.

7. Hillery, M.; O’Connell, R.F.; Scully, M.O.; Wigner, E.P. Distribution functions in physics: Fundamentals. Phys. Rep. 1984, 106, 121-167.

8. Zakos, C.K.; Fairlie, D.B.; Curtwright, T.L. Quantum Mechanics in Phase Space. An Overview with Selected Papers; World Scientific Publication: Singapore, Singapore, 2005.

9. Callender, C. Thermodynamic Asymmetry in Time. In Stanford Encyclopedia of Philosophy, Fall 2011 ed.; Zalta, E.N., Ed.; Stanford University: Stanford, CA, USA, 2011. Available online: http://plato. stanford.edu/entries/time-thermo/ (accessed on 23 February 2014).

10. Penrose, O. Foundations of statistical mechanics. Rep. Progr. Phys. 1979, 42, 1937-2006.

11. Brinkman, H.C. Brownian motion in a field of force and the diffusion theory of chemical reactions. Physica 1956, 22, 29-34.

12. Risken, H. The Fokker-Planck Equation, 2nd ed.; Springer: Berlin, Germany, 1989.

13. Coffey, W.T.; Kalmykov, Y.P. The Langevin Equation, 3rd ed.; World Scientific: Singapore, Singapore, 2012.

14. Coffey, W.T.; Kalmykov, Y.P.; Titov, S.V.; Mulligan, B.P. Wigner function approach to the quantum Brownian motion of a particle in a potential. Phys. Chem. Chem. Phys. 2007, 9, 3361-3382.

15. Alvarez-Estrada, R.F. New hierarchy for the Liouville equation, irreversibility and Fokker-Planck-like structures. Ann. Phys. 2002, 11, 357-385.

16. Alvarez-Estrada, R.F. Liouville and Fokker-Planck dynamics for classical plasmas and radiation. Ann. Phys. 2006, 15, 379-415.

17. Alvarez-Estrada, R.F. Nonequilibrium quasi-classical effective meson gas: Thermalization. Eur. Phys. J. A 2007, 31, 761-765.

18. Alvarez-Estrada, R.F. Nonequilibrium quantum anharmonic oscillator and scalar field: High temperature approximations. Ann. Phys. 2009, 18, 391-409.

19. Alvarez-Estrada, R.F. Classical systems: Moments, continued fractions, long-time approximations and irreversibility. AIP Con. Proc. 2011, 1332, 261-264.

20. Alvarez-Estrada, R.F. Classical and quantum models in nonequilibrium statistical mechanics: Moment methods and long-time approximations. Entropy 2012, 14, 291-322.

21. Alvarez-Estrada, R.F. Brownian motion, quantum corrections and a generalization of the Hermite polynomials. J. Comput. Appl. Math. 2010, 233, 1453-1461.

22. Alvarez-Estrada, R.F. Quantum Brownian motion and generalizations of the Hermite polynomials. J. Comput. Appl. Math. 2011, 236, 7-18.

23. Hochstrasser, U.W. Orthogonal Polynomials. In Handbook of Mathematical Functions; Abramowitz, M., Stegun, I.A., Eds.; Dover: New York, NY, USA, 1965. 
24. Barreiro, L.A., Campanha, J.R.; Lagos, R.E. The thermohydrodynamical picture of Brownian motion via a generalized Smoluchowski equation. Physica A 2000, 283, 160-165.

25. Doi, M; Edwards, S.F. The Theory of Polymer Dynamics; Oxford University Press: Oxford, UK, 1988.

26. Chihara, T.S. An Introduction to Orthogonal Polynomials; Gordon and Breach: New York, NY, USA, 1978.

27. Penrose, O.; Coveney, P.V. Is there a "canonical" non-equilibrium ensemble? Proc. R. Soc. Lond. 1994, A447, 631-646.

28. Beretta, G.P. Modeling non-Equilibrium dynamics of a discrete probability distribution: General rate equation for maximal entropy generation in a maximum-entropy landscape with timedependent constraints. Entropy 2008, 10, 160-182.

29. Lebon, G.; Jou, D.; Casas-Vazquez, J. Understanding Non-Equilibrium Thermodynamics; Oxford University Press: Oxford, UK, 2008.

30. Rubi, J.M. The non-equilibrium thermodynamics approach to the dynamics of mesoscopic systems. J. Non-Equilib. Thermodyn. 2004, 29, 315-326.

31. Reguera, D. Mesoscopic nonequilibrium kinetics of nucleation processes. J. Non-Equilib. Thermodyn. 2004, 29, 327-344.

32. Maes, C. Nonequilibrium entropies. Phys. Scr. 2012, 86, 058509.

33. Gyftopoulos, E.P.; Beretta, G.P. Thermodynamics. Foundations and Applications; Dover: New York, NY, USA, 2005.

34. Van Kampen, N.G. Stochastic Processes in Physics and Chemistry; Elsevier: Amsterdam, The Netherlands, 2001.

35. Gardiner, C.W.; Zoller, P. Quantum Noise, 3rd ed.; Springer: Berlin, Germany, 2004.

36. Weiss, U. Quantum Dissipative Systems, 4th ed.; World Scientific: Singapore, Singapore, 2012.

37. Breuer, H.-P.; Petruccione, F. The Theory of Open Quantum Systems; Oxford University Press: Oxford, UK, 2006.

38. Rivas, A.; Huelga, S.F. Open Quantum Systems. An Introduction; Springer: Heidelberg, Germany, 2011.

39. Hudson, R.L. When is the Wigner quasi-probability density non-negative? Rep. Math. Phys. 1974, 6, 249-252.

40. Piquet, C. Fonctions du type positif associees a deux operateurs hermitiens. C. R. Acad. Sci. Paris 1974, 279A, 107-109. (in French)

41. Schleich, W.P. Quantum Optics in Phase Space; Wiley VCH: Berlin, Germany, 2001.

42. Dahl, J.P. Springborg. The Morse oscillator in position space, momentum space and phase space. J. Chem. Phys. 1988, 88, 4535-4547.

43. Bund, G.W.; Tijero, M.C. Mapping the Wigner distribution function of the Morse oscillator into a semi-classical distribution function. 2003, arXiv:quant-ph/0304092v1.

44. Hug, M.; Menke, C.; Schleich, W.P. How to calculate the Wigner function from phase space. J. Phys. Math. Gen. 1998, 31, L217-L224.

45. Louisell, W.H. Quantum Statistical Properties of Radiation; John Wiley and Sons: New York, NY, USA, 1973. 
46. Haroche, S.; Raimond, J.-M. Exploring the Quantum; Oxford University Press: Oxford, UK, 2008.

47. Lindblad, G. On the generators of quantum dynamical semigroups. Commun. Math. Phys. 1976, 48, 119-130.

48. Huang, K. Statistical Mechanics, 2nd ed.; John Wiley and Sons: New York, NY, USA, 1987.

49. Munster, A. Statistical Thermodynamics; Springer: Berlin, Germany, 1969; Volume I.

50. Simon, B. The bound states of weakly coupled Schrödinger operators in one and two dimensions. Ann. Phys. 1976, 97, 279-288.

(c) 2014 by the author; licensee MDPI, Basel, Switzerland. This article is an open access article distributed under the terms and conditions of the Creative Commons Attribution license (http://creativecommons.org/licenses/by/3.0/). 